\title{
Cross-Correlation of Cosmic Shear and Extragalactic Gamma-ray Background: Constraints on the Dark Matter Annihilation Cross-Section
}

\author{
Masato Shirasaki \\ Department of Physics, University of Tokyo, Tokyo 113-0033, Japan* \\ Shunsaku Horiuchi \\ Center for Cosmology, Department of Physics and Astronomy, \\ 4129 Frederick Reines Hall, University of California, Irvine, CA 92697-4574t \\ Naoki Yoshida \\ Department of Physics, University of Tokyo, Tokyo 113-0033, Japan \\ Kavli Institute for the Physics and Mathematics of the Universe (WPI), \\ University of Tokyo, Kashiwa, Chiba 277-8583, Japan
}

\begin{abstract}
We present the first measurement of the cross-correlation of weak gravitational lensing and the extragalactic $\gamma$-ray background emission using data from the Canada-France-Hawaii Lensing Survey and the Fermi Large Area Telescope. The cross-correlation is a powerful probe of signatures of dark matter annihilation, because both cosmic shear and gamma-ray emission originate directly from the same DM distribution in the universe, and it can be used to derive constraints on dark matter annihilation cross-section. We show that the measured lensing- $\gamma$ correlation is consistent with a null signal. Comparing the result to theoretical predictions, we exclude dark matter annihilation cross sections of $\langle\sigma v\rangle=10^{-24}-10^{-25} \mathrm{~cm}^{3} \mathrm{~s}^{-1}$ for a $100 \mathrm{GeV}$ dark matter. If dark matter halos exist down to the mass scale of $10^{-6} M_{\odot}$, we are able to place constraints on the thermal cross sections $\langle\sigma v\rangle \sim$ $5 \times 10^{-26} \mathrm{~cm}^{3} \mathrm{~s}^{-1}$ for a $10 \mathrm{GeV}$ dark matter annihilation into $\tau^{+} \tau^{-}$. Future gravitational lensing surveys will increase sensitivity to probe annihilation cross sections of $\langle\sigma v\rangle \sim 3 \times 10^{-26} \mathrm{~cm}^{3} \mathrm{~s}^{-1}$ even for a $100 \mathrm{GeV}$ dark matter. Detailed modeling of the contributions from astrophysical sources to the cross correlation signal could further improve the constraints by $\sim 40-70 \%$.

\footnotetext{
* masato.shirasaki@utap.phys.s.u-tokyo.ac.jp

$\dagger$ s.horiuchi@uci.edu
} 


\section{INTRODUCTION}

The origin of the extragalactic gamma-ray background (EGB) emission is among the most interesting problems in astrophysics. The EGB was first detected by the $O S O-3$ satellite [1] and subsequently deduced by the $S A S-2$ satellite [2] and the Energetic Gamma-Ray Experiment Telescope onboard the Compton Gamma-ray Observatory [3]. Most recently, the Large Area Telescope (LAT) onboard the Fermi Gamma-ray Space Telescope has derived the most accurate EGB based on new data and improved modeling of the Galactic gamma-ray foreground emission. The Fermi LAT observation shows a featureless power-law spectrum for the EGB in the energy range $0.1-300 \mathrm{GeV}$ [4].

Multiple astrophysical sources of gamma rays have been proposed as contributors to the EGB. Unresolved astrophysical point sources, such as blazars and star-forming galaxies (SFG), are guaranteed sources and have been investigated by many groups. However, the modeling of the sources' faint end distributions is non-trivial, and estimates of the contribution to the EGB from unresolved blazars range from $~ 15$ per cent to $~ 100$ per cent [e.g., 5 -7]. On the other hand, the intrinsic spectral and flux properties of blazars constructed by Fermi LAT data, as well as the auto-correlation of EGB anisotropies [8], suggest that unresolved blazars can only contribute up to $\sim 20$ per cent of EGB [e.g., 9 12]. Similarly, the contribution from SFGs and radio galaxies to the EGB can be significant but is subject to large uncertainties [13, 14]. These previous works show that while the EGB intensity can be explained by the superposition of multiple astrophysical source classes, there appears to remain large uncertainties and thus, at present, an appreciable contribution from unknown or unconfirmed sources of gamma rays is allowed.

Among the potential new contributors to the EGB is the emission due to dark matter (DM) annihilation. The existence of DM is supported with high significance by a number of astrophysical observations, such as the cosmic microwave background (CMB) anisotropies [e.g., 15, 16] and large-scale structure [e.g., 17-19]. While the DM particle properties still remain unclear, if DM particles annihilate into standard model particles, as is typically expected for their production in the early universe, they will produce gamma rays that contribute to the observed EGB. The gamma-ray emission due to DM annihilation is expected to be anisotropic because of the highly non-linear gravitational growth of the DM density distribution [e.g., 20]. Although astrophysical sources are also expected to reside within DM 
halos, differences in their clustering properties may help distinguish DM annihilation signals from astrophysical contributions to the EGB.

The DM distribution in the Universe can be traced in a number of ways. Among the most powerful is gravitational lensing, which has the advantage of not requiring any assumptions such as the relation between luminosity and mass and/or hydrostatic equilibrium. The small distortions in images of distant objects caused by the large-scale matter distribution along the line of sight is called cosmic shear. The DM distribution that generate cosmic shear would also be a gamma-ray source. The cross-correlation between cosmic shear and the EGB would provide competitive information of dark matter annihilation [21]. In ref. [21], the authors also explored how astrophysical sources contribute to the cross-correlation signal, and concluded that even without detailed astrophysical modeling, the additional information derived by the cross-correlation would be helpful for a unified understanding of the EGB.

In this paper, we present the first measurement of the cross-correlation between cosmic shear and the EGB using the largest cosmic shear data set currently available from the Canada-France-Hawaii Lensing Survey (CFHTLenS) and gamma-ray photon data from the Fermi LAT telescope. We carefully remove contributions from gamma-ray point sources and the Galactic gamma-ray foreground using a likelihood modeling based on official Fermi tools and Galactic diffuse background models. We then determine the cross-correlation signal. Finally, by using statistical errors derive from the real cosmic shear and gamma-ray data, we place novel and competitive constraints on the DM annihilation cross section as functions of the DM mass and annihilation channel.

The paper is organized as follows. In Section III, we summarize the basics of DM, including the contribution to the EGB. In Section III, we describe the cosmic shear and gamma-ray data used, and provide details of the cross-correlation analysis. Our benchmark model of the cross-correlation is discussed in Section IV. In Section $\nabla$, we show the result of our crosscorrelation analysis, and discuss constraints on the DM annihilation cross section. Finally, we forecast DM constraints that can be achieved with upcoming lensing surveys. Concluding remarks and discussions are given in Section VI. Throughout, we use the standard cosmological parameters $H_{0}=100 h \mathrm{~km} \mathrm{~s}^{-1}$ with $h=0.7, \Omega_{\mathrm{m} 0}=0.279$, and $\Omega_{\Lambda}=0.721$. 


\section{DARK MATTER ANNIHILATION}

The contribution of DM annihilation to the EGB intensity $I_{\gamma}$ (the number of photons per unit energy, area, time, and solid angle) is

$$
E_{\gamma} I_{\gamma}=\frac{c}{4 \pi} \int \mathrm{d} z \frac{P_{\gamma}\left(E_{\gamma}^{\prime}, z\right)}{H(z)(1+z)^{4}} e^{-\tau\left(E_{\gamma}^{\prime}, z\right)},
$$

where $E_{\gamma}$ is the observed gamma-ray energy, $E_{\gamma}^{\prime}=(1+z) E_{\gamma}$ is the energy of the gamma ray at redshift $z, H(z)=H_{0}\left[\Omega_{\mathrm{m} 0}(1+z)^{3}+\Omega_{\Lambda}\right]^{1 / 2}$ is the Hubble parameter in a flat Universe, and the exponential factor in the integral takes into account the effect of gamma-ray attenuation during propagation owing to pair creation on diffuse extragalactic photons. Although the effect of attenuation is only important for photon energies larger than $\sim 1 \mathrm{TeV}$, and hence is not of great importance for our analysis that focuses on lower energy photons, we include it for completeness. For the gamma-ray optical depth $\tau\left(E_{\gamma}^{\prime}, z\right)$, we adopt the model in Ref. [22]. Finally, $P_{\gamma}$ is the volume emissivity (the photon energy emitted per unit volume, time, and energy range), which is given by

$$
P_{\gamma}\left(E_{\gamma}, z\right)=E_{\gamma} \frac{\mathrm{d} N_{\gamma}}{\mathrm{d} E_{\gamma}} \frac{\langle\sigma v\rangle}{2}\left[\frac{\rho_{\mathrm{dm}}(\boldsymbol{x} \mid z)}{m_{\mathrm{dm}}}\right]^{2},
$$

where $\mathrm{d} N_{\gamma} / \mathrm{d} E_{\gamma}$ is the gamma-ray spectrum per annihilation, $\langle\sigma v\rangle$ is the annihilation cross section times the relative velocity averaged with the velocity distribution function, $\rho_{\mathrm{dm}}(\boldsymbol{x} \mid z)$ is the DM mass density distribution at redshift $z$ as a function of spatial coordinate $\boldsymbol{x}$, and $m_{\mathrm{dm}}$ is the DM particle mass.

For the gamma-ray spectrum per annihilation $\mathrm{d} N_{\gamma} / \mathrm{d} E_{\gamma}$, we adopt two characteristic spectra corresponding to annihilation with $100 \%$ branching ratios to $b \bar{b}$ and $\tau^{+} \tau^{-}$final states, using the PPPC4DMID package [23] that is based on PYTHIA (v8.135) and HERWIG (v6.510) event generators. The spectra are dominated by emission from the decay of neutral pions. These are primary gamma-ray emissions, and are distinguished from secondary emission that results from interactions of the annihilation products with the environment. An example of the latter is when DM annihilation produces a positron, which, in turn, finds an electron in the galactic halo and annihilates to produce gamma rays. Also, the gamma-ray emission can be noticeably softened by the bremsstrahlung emission from leptonic final states [24]. We do not include secondary emission in this study because their effect depends strongly on the astrophysical environment and furthermore since they are only critical for annihilation 
in regions of high baryon density, e.g., the planes of galaxies. Additional contributions can arise from three-body final states such as internal bremsstrahlung [25]. This would introduce a sharp feature near the DM mass and systematically harden the gamma-ray emission. The sharp feature may enhance the correlation signal and provide a useful diagnostic for DM annihilation; it has been discussed in the context of anisotropies [26, 27]. However, we do not include this because it can only be included in the framework of a precise DM model [e.g., 23].

Since the DM annihilation rate scales with the DM density squared, highly over-dense regions such as DM halos dominate the volume emissivity. It is instructive to express the DM density $\rho_{\mathrm{dm}}$ as an overdensity $\delta(z)=\rho_{\mathrm{dm}} / \bar{\rho}_{\mathrm{dm}}(z)$ over the mean DM density $\bar{\rho}_{\mathrm{dm}}(z)=\Omega_{\mathrm{dm}} \rho_{\text {crit }}(1+z)^{3}$, where $\rho_{\text {crit }}$ is the critical density. The ensemble average of the overdensity squared, $\left\langle\delta^{2}(z)\right\rangle=\left\langle\rho_{\mathrm{dm}}^{2}(z)\right\rangle / \bar{\rho}_{\mathrm{dm}}^{2}(z)$, is called the intensity multiplier (or the clumping factor), and characterizes the enhancement in the DM annihilation rate due to dense DM halos. It is obtained by integrating over the DM halo mass function $n(M, z)$,

$$
\left\langle\delta^{2}(z)\right\rangle=\frac{1}{\bar{\rho}_{\mathrm{dm}}^{2}(z)} \int_{M_{\min }}^{\infty} \mathrm{d} M n(M, z) \int \mathrm{d} V \rho_{\mathrm{dm}}^{2}(r \mid M, z),
$$

where $\rho_{\mathrm{dm}}(r \mid M, z)$ describes the density profile as a function of radius $r$ for a DM halo with mass $M$ at redshift $z$, and $M_{\min }$ is the smallest DM halo mass.

Estimates of the flux multiplier depend on the value of $M_{\min }$, the halo mass function, the DM density profile, and how the DM profile depend on halo mass and evolve in redshift. Among these, the value of $M_{\min }$ has the largest impact. The smallest DM halo mass ought to be determined from the DM particle properties, being the Jeans mass of dark matter particles. For supersymmetric neutralinos and $\sim \mathrm{MeV} \mathrm{DM}$, this is some $10^{-6} M_{\odot}$ [28], while other DM particles have $M_{\text {min }}$ that vary by orders of magnitudes [29 31]. However, complications arise because not all DM halos survive the process of mergers and tidal interactions during structure formation. In particular, much of the smallest DM halos may be absorbed into larger halos and their central densities disrupted before they appreciably contribute to the EGB [e.g., 32, 33]. The DM Jeans mass is therefore simply a lower limit. Furthermore, for secondary gamma-ray emission, the relevant minimum mass is set by the Jeans mass of the baryons, which is on the order of $\sim 10^{6} M_{\odot}$ [e.g., 28]. In Section IVB, we discuss the details of the calculation of the flux multiplier in the halo model approach, and also discuss the effects of substructures residing within halos. 
Using the flux multiplier, the contribution to the EGB is

$$
I_{\gamma}=\left.\frac{\langle\sigma v\rangle}{8 \pi} \int c \mathrm{~d} z \frac{d N_{\gamma}}{d E_{\gamma}}\right|_{E_{\gamma}^{\prime}} \frac{e^{-\tau\left(E_{\gamma}^{\prime}, z\right)}}{H(z)(1+z)^{3}}\left(\frac{\bar{\rho}_{\mathrm{dm}}(z)}{m_{\mathrm{dm}}}\right)^{2}\left\langle\delta^{2}(z)\right\rangle,
$$

where the particle properties of $\mathrm{DM}-m_{\mathrm{dm}},\langle\sigma v\rangle$, and $\mathrm{d} N_{\gamma} / \mathrm{d} E_{\gamma}-$ are conveniently decoupled from the physics determining its spatial distribution, $\left\langle\delta^{2}(z)\right\rangle$.

\section{DATA}

\section{A. Cosmic shear data}

We use the cosmic shear data from the Canada-France-Hawaii Telescope Lensing Survey [CFHTLenS; 34]. CFHTLenS is a 154 square deg multi-color optical survey in five optical bands $u^{*}, g^{\prime}, r^{\prime}, i^{\prime}, z^{\prime}$. CFHTLenS is optimized for weak lensing analysis with a full multicolor depth of $i_{A B}^{\prime}=24.7$ with optimal sub-arcsec seeing conditions. The survey consists of four separated fields called W1, W2, W3, and W4, with an area of $\sim 72,30,50$, and 25 square degs, respectively.

The CFHTLenS survey analysis mainly consists of the following three processes: photometric redshift measurement [35], weak lensing data processing with THELI [36], and shear measurement with lensfit [37]. A detailed systematic error study of the shear measurements in combination with the photometric redshifts is presented in Ref. [34] and additional error analyses of the photometric redshift measurements are presented in Ref. [38].

The ellipticities of the source galaxies in the data have been calculated using the lensfit algorithm. lensfit performs a Bayesian model fitting to the imaging data by varying a galaxy's ellipticity and size, and by marginalizing over the centroid position. It adopts a forward convolution process that convolves the galaxy model with the point spread function (PSF) to estimate the posterior probability of the model given the data. For each galaxy, the ellipticity $\epsilon$ is estimated as the mean likelihood of the model posterior probability after marginalizing over galaxy size, centroid position, and bulge fraction. An inverse variance weight $w$ is given by the variance of the ellipticity likelihood surface and the variance of the ellipticity distribution of the galaxy population. The lensfit algorithm has been tested with image simulations in detail. The observed ellipticities $\boldsymbol{\epsilon}^{\text {obs }}$ with any shape measurement 
method are calibrated in practice as

$$
\boldsymbol{\epsilon}^{\mathrm{obs}}=(1+m) \boldsymbol{\epsilon}^{\mathrm{true}}+\boldsymbol{c}
$$

where $m$ is a multiplicative bias and $\boldsymbol{c}$ is an additive bias. In the case of lensfit, $\boldsymbol{c}$ is consistent with zero for a large set of simulated images but $m$ cannot be negligible and it depends on both galaxy signal-to-noise ratio and size. On a weight average, this multiplicative bias corresponds to a $6 \%$ correction. In terms of statistical quantities such as two point correlation function, this bias is easily corrected by multiplying an overall factor (see Ref. [37] for further details).

The PSF in optical imaging surveys is one of the major systematics of galaxy shape measurement. The optical PSF originates from diffraction, the atmospheric turbulence, optical aberration, the misalignment of CCD chips on a focal plane, and pixelization effects. Anisotropy of the PSF causes a coherent deformation of images that might mimic the tangential shear pattern due to large scale structure in the universe. Often in cosmic shear measurement, systematic effects are tested through statistical analyses of the $45^{\circ}$ rotated component of galaxy ellipticities. This is because the $45^{\circ}$ rotated component of cosmic shear should vanish statistically. In Section $\mathrm{V}$, we perform statistical analysis by using the $45^{\circ}$ rotated component of galaxy ellipticities and we quantify systematics, if any, of the lensing data set.

The photometric redshifts $z_{p}$ are estimated by the BPZ code [39, Bayesian Photometric Redshift Estimation]. The true redshift distribution is well described by the sum of the probability distribution functions (PDFs) estimated from BPZ [38]. The galaxy-galaxy-lensing redshift scaling analysis confirms that contamination is not significant for galaxies selected at $0.2<z_{p}<1.3$ [34]. In this redshift range, the weighted median redshift is $\sim 0.7$ and the effective weighted number density $n_{\text {eff }}$ is 11 per square arcmin. We have used the source galaxies with $0.2<z_{p}<1.3$ to measure the cross-correlation of cosmic shear and EGB presented in Section IV. We use a total of 2570270, 679070, 1649718, and 770356 galaxies in the W1, W2, W3, and W4 fields, respectively, for our cross-correlation study.

\section{B. Extragalactic gamma-ray background data}

We use Fermi-LAT Pass 7 Reprocessed gamma-ray photon data taken from August 2008 to January 2014. For each CFHTLenS patch, we download photons within a circle of radius 
$10^{\circ}$ around the center of each region and work with a $14^{\circ} \times 14^{\circ}$ square region of interest $(\mathrm{ROI})$. We use the Fermi Tools version v9r32p5 to analyze the datal. Using the gtmktime tool, we remove data taken during non-survey modes and when the satellite rocking angle exceeds $52^{\circ}$ with respect to the zenith (DATA_QUAL=1, LAT_CONFIG=1, and ABS (ROCK_ANGLE) <52). This standard procedure removes epochs with potentially significant contamination by the gamma-ray bright Earth limb. Unless otherwise stated, we work with only ULTRACLEAN-class photons, which are events that pass the most stringent quality cuts, and we use photons between 1-500 GeV in energy. In Section IVA, we discuss using SOURCE-class photons. We use the gtbin tool to bin the photons in a stereographic projection into pixels of $0.2^{\circ} \times 0.2^{\circ}$ and into 30 equal logarithmically-spaced energy bins. These binning sizes are taken from the official recommended values that are chosen to ensure reasonable analysis outcomes, namely, to ensure that rapid variations of the effective area with energy is taken into account (e.g., as discussed in the binned likelihood tutorial of the Fermi Analysis Threads). With the data selection cuts in place, we use the gtltcube tool to generate integrated live times and the gtexpсube2 tool to generate the integrated exposure maps. Throughout, we work with the P7REP_ULTRACLEAN_V15 instrument response function (IRF), unless otherwise stated.

In order to obtain the extragalactic diffuse photons, for each ROI we subtract the best fit Galactic foreground emission model from the raw data. We then mask out point sources using a mask of $2^{\circ}$ radius around each point source. The mask size corresponds to a generous estimate of the PSF of the Fermi-LAT detector, which decreases with energy: the $68 \%$ containment angle is $\sim 0.9 \mathrm{deg}$ at $1 \mathrm{GeV}$ and $\sim 0.26 \mathrm{deg}$ at $10 \mathrm{GeV}$, both for combined front and back conversion tracks. Since most point sources have steep spectra and hence dominated by low-energy photons, our adopted mask is chosen to be sufficiently larger than the containment angle at our lower energy limit of $1 \mathrm{GeV}$. We discuss the potential of smaller mask sizes in Section VI.

The best fit Galactic diffuse emission model is estimated separately for each ROI, by including all the point sources in the ROI in the 2FGL catalog, together with the recommended Galactic diffuse emission model (gll_iem_v05) and the recommended isotropic emission model (iso_clean_v05). We have checked that our four ROIs are sufficiently far from the large-scale diffuse gamma-ray sources such as the Fermi bubbles [40] which would otherwise complicate fitting. The CFHTLenS patches each have 9, 11, 11, and 12 point

\footnotetext{
${ }^{1}$ http://fermi.gsfc.nasa.gov/ssc/data/analysis/
} 
sources, respectively. We use the gtlike tool to perform a binned likelihood analysis, varying all point source spectra as well as the diffuse emission normalizations. We then use the gtmodel tool to generate photon counts maps based on the best fit Galactic diffuse model and exposure maps. Finally, we subtract these from the raw counts maps. We checked that the procedure yields a flux spectrum for the EGB, estimated as the raw counts minus a model without the isotropic component, divided by the exposure map, that is very similar to the -2.41 power-law spectrum of the EGB reported in Ref. [4]. In Figure 1, we show how the residuals of the raw counts minus the Galactic diffuse model, demonstrate structureless spatial maps in all four CFHTLenS fields.

\section{CROSS-CORRELATION OF COSMIC SHEAR AND EGB}

\section{A. Analysis}

In order to calculate the cross-correlation of cosmic shear and EGB, we use the following estimator:

$$
\xi_{\delta n-\gamma_{t}}(\theta)=\frac{\sum_{i}^{N_{\text {pixel }}} \sum_{j}^{N_{\text {gal }}}\left(n^{\mathrm{obs}}\left(\boldsymbol{\phi}_{i}\right)-n^{\mathrm{gm}}\left(\boldsymbol{\phi}_{i}\right)\right) w_{j} \epsilon_{t}\left(\boldsymbol{\phi}_{j} \mid \boldsymbol{\phi}_{i}\right) \Delta_{\theta}\left(\boldsymbol{\phi}_{i}-\boldsymbol{\phi}_{j}\right)}{(1+K(\theta)) \sum_{i}^{N_{\text {pixel }}} \sum_{j}^{N_{\text {gal }}} w_{j} \Delta_{\theta}\left(\boldsymbol{\phi}_{i}-\boldsymbol{\phi}_{j}\right)}
$$

where $N_{\text {pix }}$ is the number of pixels in the gamma-ray counts map, $N_{\text {gal }}$ is the number of galaxies, $n^{\text {obs }}\left(\phi_{i}\right)$ is the observed number of photons in pixel $i$ in the gamma-ray counts map, $n^{\mathrm{gm}}\left(\boldsymbol{\phi}_{i}\right)$ is the contribution from the Galactic emission model estimated using the Fermi-LAT diffuse template and detector modeling, $w_{j}$ is the weight related to the shape measurement, and $\epsilon_{t}\left(\boldsymbol{\phi}_{j} \mid \boldsymbol{\phi}_{i}\right)$ is the tangential component of the $j$-th galaxy's ellipticity with respect to the $i$-th pixel of the gamma-ray counts map, defined by

$$
\epsilon_{t}\left(\phi_{j} \mid \phi_{i}\right)=-\epsilon_{1}\left(\phi_{j}\right) \cos \left(2 \alpha_{i j}\right)-\epsilon_{2}\left(\phi_{j}\right) \sin \left(2 \alpha_{i j}\right)
$$

where $\alpha_{i j}$ is defined as the angle measured from the right ascension direction to a line connecting the $i$-th pixel and the $j$-th galaxy. We define the function $\Delta_{\theta}(\phi)=1$ for $\theta-$ $\Delta \theta / 2 \leq \phi \leq \theta+\Delta \theta / 2$ and zero otherwise. The overall factor $1+K(\theta)$ in Eq. ([6]) is used to correct for the multiplicative shear bias $m$ in the shape measurement with lensfit [37], 

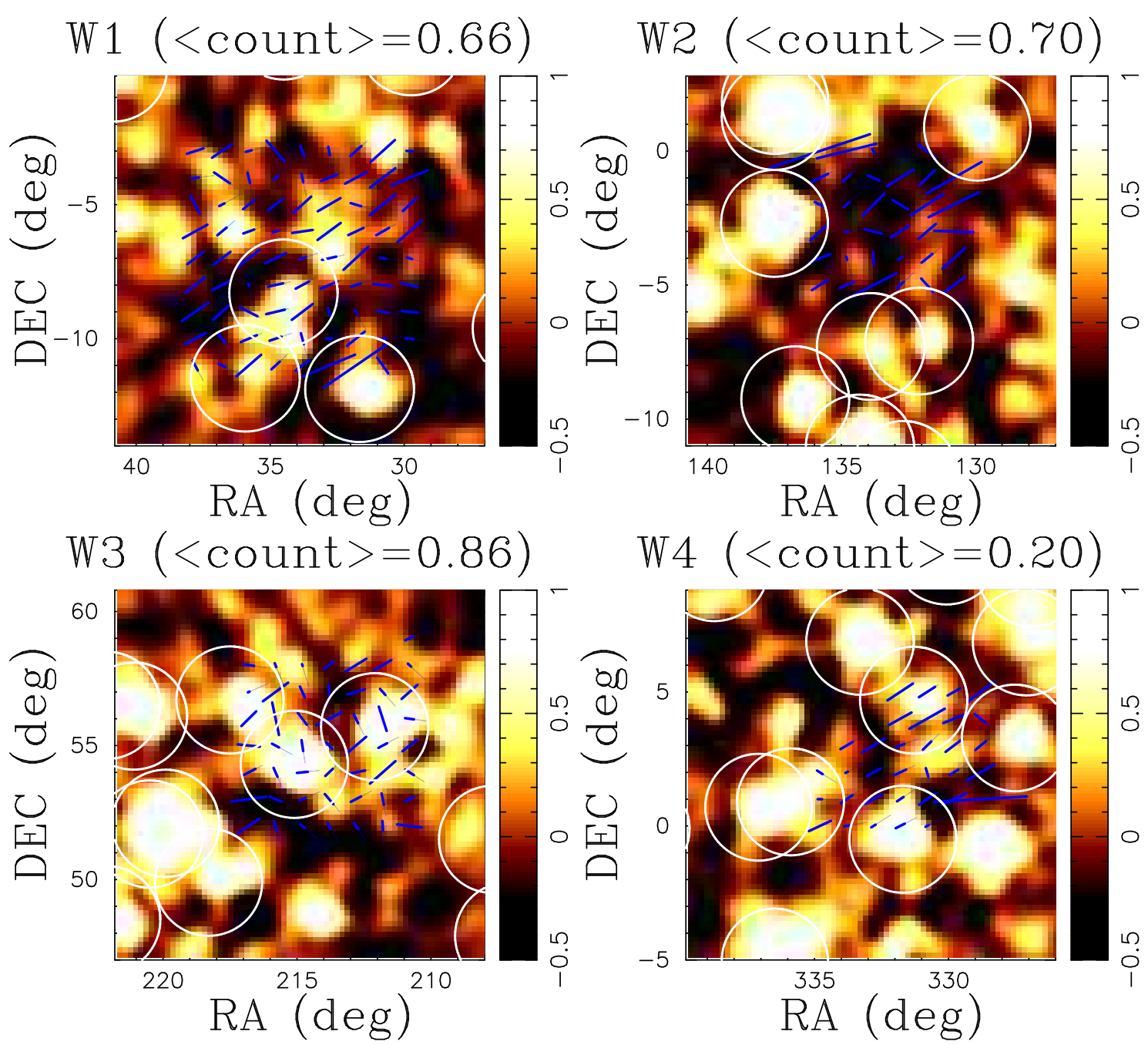

W4 $(<$ count $>=0.20)$

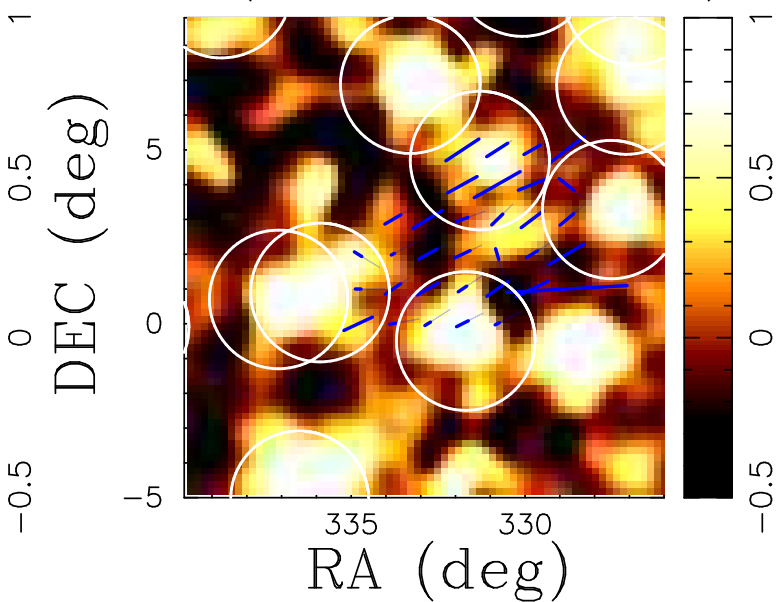

FIG. 1. Residual maps in the CFHTLenS W1, W2, W3, and W4 fields, where residual is defined as the fluctuation in the EGB photon count map from its mean value. In each panel, the color-scale bar shows both the positive and negative difference between the EGB count map and the mean of each field indicated above the panels: $0.66,0.70,0.86$, and 0.20 in W1, W2, W3 and W4 fields, respectively. Overlaid by thick lines are the average ellipticities of source galaxies over $1 \operatorname{deg}^{2}$ with arbitrary scaling. The circles represent the point-source masked regions. For visualization purposes, a Gaussian smoothing is performed on the map with a width of $0.6 \mathrm{deg}$. 
which is given by

$$
1+K(\theta)=\frac{\sum_{i}^{N_{\text {pixel }}} \sum_{j}^{N_{\text {gal }}} w_{j}\left(1+m\left(\boldsymbol{\phi}_{j}\right)\right) \Delta_{\theta}\left(\boldsymbol{\phi}_{i}-\boldsymbol{\phi}_{j}\right)}{\sum_{i}^{N_{\text {pixel }}} \sum_{j}^{N_{\text {gal }}} w_{j} \Delta_{\theta}\left(\boldsymbol{\phi}_{i}-\boldsymbol{\phi}_{j}\right)} .
$$

We have checked that our estimator is consistent with a zero signal when applied to randomized shear catalogues and the observed photon count map. We have also tested a combination of random photon count map with the observed shear catalogue.

For binning in angular separation $\theta$, we set the innermost separation bin to 1 arcmin and use 10 bins logarithmically spaced in $\Delta \log _{10} \theta=0.2$. In calculating Eq. (66), we do not perform pixelization in the galaxy catalogue. We simply consider the center of each pixel in the gamma-ray map as the angular position of the gamma-ray photons to perform the summation in Eq. (6) . To be precise, this induces an artificial smoothing over smaller scales than the pixel size in our gamma-ray map, i.e., 0.2 deg. However, we do not expect to detect physically important correlations over such small angular scales due to blurring by the PSF of the Fermi-LAT detector, as we show in Section IVB. In the present paper, we take the PSF smearing into account in theoretical models (see Figure 4). Note that the pixelization effect in the gamma-ray map is included in the covariance of our estimator. The pixelization effect is found to be unimportant in detection of the cross-correlation signals at large angular separations.

The statistical properties of our estimator Eq. (므) are summarized in Appendix A. There, we present the exact formulation of the covariance of our estimator and derive two dominant contributions; they arise from the intrinsic shape variance of galaxies, called shape noise, and the finite number of photon counts per pixel in the gamma-ray maps, called photon noise. We use randomized shear catalogues in order to estimate the statistical errors associated with the shape noise. To this end, we generate 500 randomized shear catalogues by rotating the direction of each galaxy ellipticity but with fixed amplitude [41]. We then estimate the covariance matrix $C_{i j}$ of the estimator Eq. (마) by

$$
C_{i j}=\frac{1}{N_{\mathrm{re}}-1} \sum_{r}\left(\xi_{\delta n-\gamma_{t}}^{r}\left(\theta_{i}\right)-\bar{\xi}_{\delta n-\gamma_{t}}\left(\theta_{i}\right)\right)\left(\xi_{\delta n-\gamma_{t}}^{r}\left(\theta_{j}\right)-\bar{\xi}_{\delta n-\gamma_{t}}\left(\theta_{j}\right)\right)
$$

where $\xi_{\delta n-\gamma_{t}}^{r}\left(\theta_{i}\right)$ is the estimator for the $i$-th angular bin obtained from the $r$-th realization, and $N_{\text {re }}=500$ is the number of randomized catalogues. The ensemble average of the $i$-th 


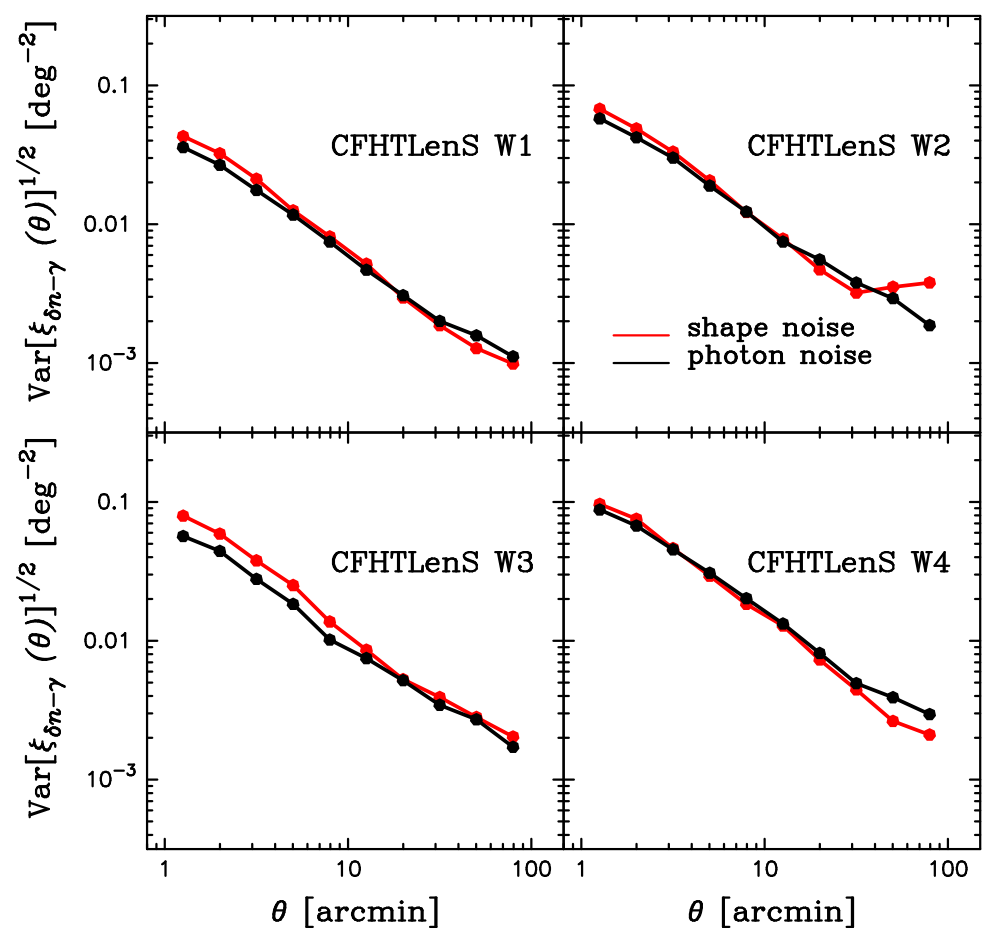

FIG. 2. The variance of cross-correlation signals estimated from a set of randomized realizations and the observed map. The red line in each panel represents the statistical error associated with the shape measurement. The black line shows the statistical error associated with the Poisson error from the finite number of gamma-ray counts.

angular bin over 500 realizations, $\bar{\xi}_{\delta n-\gamma_{t}}\left(\theta_{i}\right)$, is simply given by

$$
\bar{\xi}_{\delta n-\gamma_{t}}\left(\theta_{i}\right)=\frac{1}{N_{\mathrm{re}}} \sum_{r} \xi_{\delta n-\gamma_{t}}^{r}\left(\theta_{i}\right)
$$

To simulate the photon count noise, we generate 500 randomized count maps assuming the photon counts in each pixel follows a Poisson distribution with a mean of $n^{\text {obs }}(\phi)$. We repeat the cross-correlation analysis with the 500 count maps and the observed galaxy shear catalogue. We then estimate the statistical error related to the photon noise in the same manner shown in Eq. (9). In total, we estimate the statistical error associated with the shape measurement and the photon noise by summing these two contributions. Figure 2 shows the variance of the cross-correlation signal estimated from the two sets of randomized realizations as described above. In each panel, the red line shows the contribution from the shape noise and the black line shows the variance due to the photon noise. Overall, the shape noise and the photon noise contribute to the statistical error of our estimator at 
similar levels.

The cross-correlation estimator is also dependent on the model for the foreground astrophysical diffuse emission of our own Galaxy. We therefore investigate alternate LAT diffuse models provided by the Fermi collaboration to assess differences in the estimated EGB photons. First we work with Fermi LAT Pass 7 reprocessed SOURCE-class photons, which is made with a weaker set of cuts to remove cosmic-ray induced backgrounds, and analyze them adopting the appropriate diffuse model and IRF. Second, we work with the Fermi LAT Pass 7 photon pipeline rather than Pass 7 reprocessed photons with respectively the appropriate diffuse emission model (gal_2yearp7v6_v0 and iso_p7v6clean) and IRF. In both cases, we first find the best fit diffuse model normalizations, subtract the best fit Galactic diffuse maps from the raw data, and then mask the point sources, to obtain finally the EGB photons. We have explicitly checked that the different Galactic diffuse models do not significantly affect our cross-correlation analyses. We discuss this issue later in Section VI.

It may be necessary to consider another important contribution to the covariance, i.e., the sampling variance. To estimate the sampling variance, one could use the halo model approach IVB, but it is uncertain how the astrophysical sources are included in the model. Because we expect the sampling variance to be less important compared to the uncertainty of the halo model itself, we simply ignore the sampling variance but include the model uncertainty as presented in IVB when deriving the constraints on DM annihilation.

\section{B. Theoretical model}

In this section, we summarize our benchmark model for the cross-correlation signal between cosmic shear and the EGB. The theoretical framework for the angular power spectrum analysis of the EGB has been developed in Refs. [20, 21, 42, 43]. We calculate the crosscorrelation of cosmic shear and the EGB as follows.

In general, the number of EGB photons along the line of sight $\boldsymbol{\theta}$ can be expressed by

$$
\delta n(\boldsymbol{\theta})=\int \mathrm{d} \chi g(\chi, \boldsymbol{\theta}) W_{g}(\chi)
$$

where $\chi$ is the comoving distance, $g$ is the relevant field for gamma-ray sources, and $W_{g}$ is the window function. In the case of gamma-ray emission from DM annihilation, the relevant 
field is the overdensity squared $\delta^{2}$, and the window function is given by

$$
W_{g}(\chi)=\left.\int_{E_{\gamma, \min }}^{E_{\gamma, \max }} \mathrm{d} E_{\gamma} \frac{\langle\sigma v\rangle}{8 \pi}\left(\frac{\bar{\rho}_{\mathrm{dm}, 0}}{m_{\mathrm{dm}}}\right)^{2}[1+z(\chi)]^{3} \frac{\mathrm{d} N_{\gamma}}{\mathrm{d} E_{\gamma}}\right|_{E_{\gamma}^{\prime}} \exp \left[-\tau\left(E_{\gamma}^{\prime}, \chi\right)\right] \eta\left(E_{\gamma}\right),
$$

where $\bar{\rho}_{\mathrm{dm}, 0}$ is the mean density of DM at present, $E_{\gamma}^{\prime}=(1+z(\chi)) E_{\gamma}$ and $E_{\gamma}$ are the energy of the gamma ray when it is emitted at $\chi$ and when it is observed, respectively, and $\eta\left(E_{\gamma}\right)$ is the exposure which is the integral of effective area over time taking into account the orbits of Fermi and data cuts. We use a standard model of $\tau$ [22], and we estimate $\eta\left(E_{\gamma}\right)$ by averaging the exposure maps over the ROI in each of the CFHTLenS patches.

We next consider gravitational lensing by large-scale structure. When one denotes the observed position of a source object as $\boldsymbol{\theta}$ and the true position as $\boldsymbol{\beta}$, one can characterize the distortion of image of a source object by the following $2 \mathrm{D}$ matrix:

$$
A_{i j}=\frac{\partial \beta^{i}}{\partial \theta^{j}} \equiv\left(\begin{array}{cc}
1-\kappa-\gamma_{1} & -\gamma_{2} \\
-\gamma_{2} & 1-\kappa+\gamma_{1}
\end{array}\right)
$$

where $\kappa$ is convergence and $\gamma$ is shear. In the weak lensing regime (i.e., $\kappa, \gamma \ll 1$ ), each component of $A_{i j}$ can be related to the second derivative of the gravitational potential $\Phi$ as

$$
\begin{aligned}
A_{i j} & =\delta_{i j}-\Phi_{i j}, \\
\Phi_{i j} & =\frac{2}{c^{2}} \int_{0}^{\chi} \mathrm{d} \chi^{\prime} f\left(\chi, \chi^{\prime}\right) \frac{\partial^{2}}{\partial x_{i} \partial x_{j}} \Phi\left[r\left(\chi^{\prime}\right) \boldsymbol{\theta}, \chi^{\prime}\right], \\
f\left(\chi, \chi^{\prime}\right) & =\frac{r\left(\chi-\chi^{\prime}\right) r\left(\chi^{\prime}\right)}{r(\chi)}
\end{aligned}
$$

where $r(\chi)$ is angular diameter distance, and $x_{i}=r \theta_{i}$ represents physical distance [44, 45]. By using the Poisson equation, one can relate the convergence field to the matter overdensity field $\delta$ [44, 45]. Weak lensing convergence field is then given by

$$
\kappa(\boldsymbol{\theta}, \chi)=\frac{3}{2}\left(\frac{H_{0}}{c}\right)^{2} \Omega_{\mathrm{m} 0} \int_{0}^{\chi} \mathrm{d} \chi^{\prime} f\left(\chi, \chi^{\prime}\right) \frac{\delta\left[r\left(\chi^{\prime}\right) \boldsymbol{\theta}, \chi^{\prime}\right]}{a\left(\chi^{\prime}\right)} .
$$

Because source galaxies are distributed over a range of redshift, we denote the source distribution by $p(\chi)$. In this case, convergence field on the $\boldsymbol{\theta}$ coordinate is expressed as

$$
\kappa(\boldsymbol{\theta})=\int \mathrm{d} \chi W_{\kappa}(\chi) \delta(\boldsymbol{\theta}, \chi),
$$

where window function for $\kappa$ is given by

$$
W_{\kappa}(\chi)=\frac{3}{2}\left(\frac{H_{0}}{c}\right)^{2} \Omega_{\mathrm{m} 0}(1+z(\chi)) \int_{\chi}^{\infty} \mathrm{d} \chi^{\prime} p\left(\chi^{\prime}\right) f\left(\chi^{\prime}, \chi\right) .
$$


In this paper, for $p(\chi)$, we use the sum of the posterior probability distribution function of photometric redshift [19, 41].

Using Eqs. (11) and (18) with Limber approximation [46, 47], we obtain the angular cross power spectrum of $\delta n$ and $\kappa$ as

$$
P_{\delta n-\kappa}(\ell)=\int \frac{\mathrm{d} \chi}{\chi^{2}} W_{g}(\chi) W_{\kappa}(\chi) P_{\delta-\delta^{2}}(\ell / \chi, z(\chi)) .
$$

The direct observable in the present study is the cross-correlation function in real space, which is calculated as

$$
\xi_{\delta n-\gamma_{t}}(\theta)=\int \frac{\mathrm{d} \ell \ell}{2 \pi} P_{\delta n-\kappa}(\ell) J_{2}(\ell \theta)
$$

where $J_{2}(x)$ represents the second-order Bessel function [48, 49].

The integrand $P_{\delta-\delta^{2}}(k, z)$ in Eq. (20) is calculated by following the so-called halo model approach [50]. The halo model is a useful approach for incorporating the non-linear growth of the overdensity $\delta$ that determines the anisotropy of the EGB. With the halo model approach, $P_{\delta-\delta^{2}}(k, z)$ can be expressed as a sum of two terms called the one-halo term and the twohalo term. The former represents the two-point correlation within a given DM halo, and the latter corresponds to the correlation due to clustering of DM haloes. These two terms can be written as, respectively,

$$
\begin{aligned}
P_{\delta-\delta^{2}}^{1 h}(k, z)= & \left(\frac{1}{\bar{\rho}_{m}}\right)^{3} \int_{M_{\min }} \mathrm{d} M n(M, z) M u(k \mid M, z) \\
& \times\left(1+b_{s h}(M)\right) v(k \mid M, z) \int \mathrm{d} V \rho_{h}^{2}(r \mid M, z) \\
P_{\delta-\delta^{2}}^{2 h}(k, z)= & P^{\operatorname{lin}}(k, z)\left(\frac{1}{\bar{\rho}_{m}}\right)^{3}\left[\int_{M_{\min }} \mathrm{d} M n(M, z) b_{h}(M, z) M u(k \mid M, z)\right] \\
& \times\left[\int_{M_{\text {min }}} \mathrm{d} M n(M, z) b_{h}(M, z)\left(1+b_{s h}(M)\right) v(k \mid M, z) \int \mathrm{d} V \rho_{h}^{2}(r \mid M, z)\right]
\end{aligned}
$$

where $n(M, z)$ is the halo mass function, and $b_{h}(M, z)$ is the linear halo bias [51, 52]. We adopt the Navarro-Frenk-White (NFW) DM density profile [53],

$$
\rho_{h}(r \mid M, z)=\frac{\rho_{s}}{\left(r / r_{s}\right)\left(1+r / r_{s}\right)^{2}}
$$

where $\rho_{s}$ and $r_{s}$ are the scale density and the scale radius, respectively. These parameters can be condensed into one parameter, the concentration $c_{\mathrm{vir}}(M, z)$, by the use of two halo mass relations; namely, $M=4 \pi r_{\text {vir }}^{3} \Delta_{\text {vir }}(z) \rho_{\text {crit }}(z) / 3$, where $r_{\text {vir }}$ is the virial radius corresponding to the overdensity criterion $\Delta_{\mathrm{vir}}(z)$ as shown, e.g., in Ref. [54], and $M=\int d V \rho_{h}\left(\rho_{s}, r_{s}\right)$ 
with the integral performed out to $r_{\text {vir. }}$. In this paper, we adopt the functional form of the concentration parameter in Ref. [55]. The volume integral of the density squared with Eq. (24) is then

$$
\int \mathrm{d} V \rho_{h}^{2}(r \mid M, z)=\frac{4 \pi r_{s}^{3} \rho_{s}^{2}}{3}\left[1-\frac{1}{\left(1+c_{\mathrm{vir}}\right)^{3}}\right] .
$$

$u(k \mid M, z)$ and $v(k \mid M, z)$ represent the Fourier transform of density profile and density squared profile, respectively. Both $u(k \mid M, z)$ and $v(k \mid M, z)$ are normalized so as to become unity in the limit of $k \rightarrow 0$. We use the Fourier transform of normalized NFW profile for $u(k \mid M, z)$ as given in Ref. [50], and the functional form of $v(k \mid M, z)$ in Ref. [43]. Finally, $b_{s h}$ is the boost factor, which is essentially equal to the flux multiplier $\left\langle\delta^{2}(z)\right\rangle$. However, in addition to the contribution from DM halos described in Section II, subhalos that reside within halos similarly boost the DM annihilation rate. We adopt the fitting formula for $b_{s h}$ provided by Ref. [56] that includes this extra effect. Based on recent high-resolution dissipationless $N$-body numerical simulations, they find that $b_{s h}=1.6 \times 10^{-3}\left(M / M_{\odot}\right)^{0.39}$ provides a satisfactory fit.

The minimum halo mass $M_{\min }$ in Eqs. (22) and (23) is one of the largest model uncertainties. As discussed in Section [II, it has a large range of possibilities. For the purposes of our analysis, we consider two cases: a conservative case with $M_{\min }=10^{6} M_{\odot}$ that corresponds to the typical baryonic Jeans mass [28], and an optimistic case with $M_{\min }=10^{-6} M_{\odot}$ which is the typical free streaming scale for neutralino DM. In our benchmark model, the difference in $M_{\min }$ changes the amplitude of cross-correlation signal $\xi_{\delta n-\gamma_{t}}(\theta)$ by a factor of $\sim 10$. We regard this variation as our model uncertainty. Namely, the uncertainty of our benchmark model is a factor of $\sim 10$. Note that this model uncertainty likely dominates over the systematic uncertainties in the Galactic diffuse template and those due to sample variance in our weak lensing shear measurement.

It has recently been argued that the halo profile concentration shows a peculiar dependence on the halo mass, and that the simple power-law extrapolation for concentration used in Ref. [56] results in an overestimate of the boost factor by a factor of $\sim 50$ depending on $M_{\min }$ [e.g., 57, 58]. Because most of the cross-correlation signal comes from clustering at large angular scales (see Figure 8 later in Section $\overline{\nabla A}$ ), our results are not strongly affected by the choice. We discuss this point further in detail in Appendix B, 


\section{Astrophysical source contribution}

Astrophysical sources such as blazars and SFGs contribute to the EGB. We calculate the contribution to $P_{\delta n-\kappa}(\ell)$ as

$$
P_{\delta n-\kappa}(\ell)=\int \frac{\mathrm{d} \chi}{\chi^{2}} W_{g, \text { ast }}(\chi) W_{\kappa}(\chi) P_{\delta-L}(\ell / \chi, z(\chi)),
$$

where $W_{g \text {,ast }}(\chi)$ represents the window function of gamma rays from astrophysical sources, and $P_{\delta-L}(k, z)$ is the three dimensional cross power spectrum of matter over density and luminosity. The weight function $W_{g \text {,ast }}$ is given by

$$
W_{g, \text { ast }}(\chi)=\int_{E_{\min }}^{E_{\max }} \frac{\mathrm{d} E_{\gamma}}{4 \pi} N_{0}(\chi)\left(\frac{E_{\gamma}^{\prime}}{E_{0}}\right)^{-\alpha} \exp \left[-\tau\left(E_{\gamma}^{\prime}, \chi\right)\right] \eta\left(E_{\gamma}\right),
$$

where $E_{0}=100 \mathrm{MeV}, E_{\gamma}^{\prime}=(1+z(\chi)) E_{\gamma}$, and $N_{0}(\chi)\left(E_{\gamma} / E_{0}\right)^{-\alpha}$ represents the gamma-ray energy distribution of the astrophysical sources. In modeling $P_{\delta-L}$, one can use a similar formalism to Eqs. (22) and (23) but replacing the mass function $n(M, z) \mathrm{d} M$ by the lumi-

nosity function $\Phi(L, z) \mathrm{d} L$ [21]. Assuming blazars and SFGs are well approximated as point sources, $P_{\delta-L}$ can be divided into two terms,

$$
\begin{aligned}
P_{\delta-L}^{1 h}(k, z)= & \frac{1}{\bar{\rho}_{m}\langle L\rangle(\chi)} \int_{L_{\min }(z)}^{L_{\max }(z)} \mathrm{d} L \Phi(L, z) L u(k \mid M(L), z) \\
P_{\delta-L}^{2 h}(k, z)= & P^{\operatorname{lin}}(k, z)\left(\frac{1}{\bar{\rho}_{m}\langle L\rangle(\chi)}\right)\left[\int_{M_{\min }} \mathrm{d} M n(M, z) b_{h}(M, z) u(k \mid M, z)\right] \\
& \times \int_{L_{\min }(z)}^{L_{\max }(z)} \mathrm{d} L \Phi(L, z) L b_{h}(M(L), z),
\end{aligned}
$$

where $\langle L\rangle(\chi)$ is the mean luminosity at $z(\chi)$ and $M(L)$ is the mass-luminosity relation of astrophysical sources. We therefore need to set the specific functional form of $N_{0}(\chi), \Phi(L, z)$, $M(L)$, and the power-law index of energy distribution of gamma-ray $\alpha$ in order to calculate $P_{\delta n-\kappa}(\ell)$ for each astrophysical source.

For the gamma-ray luminosity function of blazars, we adopt the luminosity-dependent density evolution model [6, 42] with parameters in Ref. [43]. We set the power law index $\alpha$ for blazars to be 2.4 , which is consistent with the spectra of resolved blazars. The gamma-ray luminosity of blazars is evaluated as $\nu L_{\nu}$ at $100 \mathrm{MeV}$. In this case, $N_{0}$ is given by $\langle L\rangle / E_{0}^{2}$. We adopt the mass-luminosity relation $M(L)=10^{11.3} M_{\odot}\left(L / 10^{44.7} \mathrm{erg} \mathrm{s}^{-1}\right)^{1.7}$ that yields the desired bias of blazer host halos [42]. We assume that there are no blazars fainter than the luminosity $L_{\min }=10^{42} \mathrm{erg} \mathrm{s}^{-1}$ at any redshift. In estimating $L_{\max }(z)$, we assume a blazar can be resolved if the gamma-ray flux $F$ at $E>100 \mathrm{MeV}$ is larger than $2 \times 10^{-9} \mathrm{~cm}^{-2} \mathrm{~s}^{-1}$. 


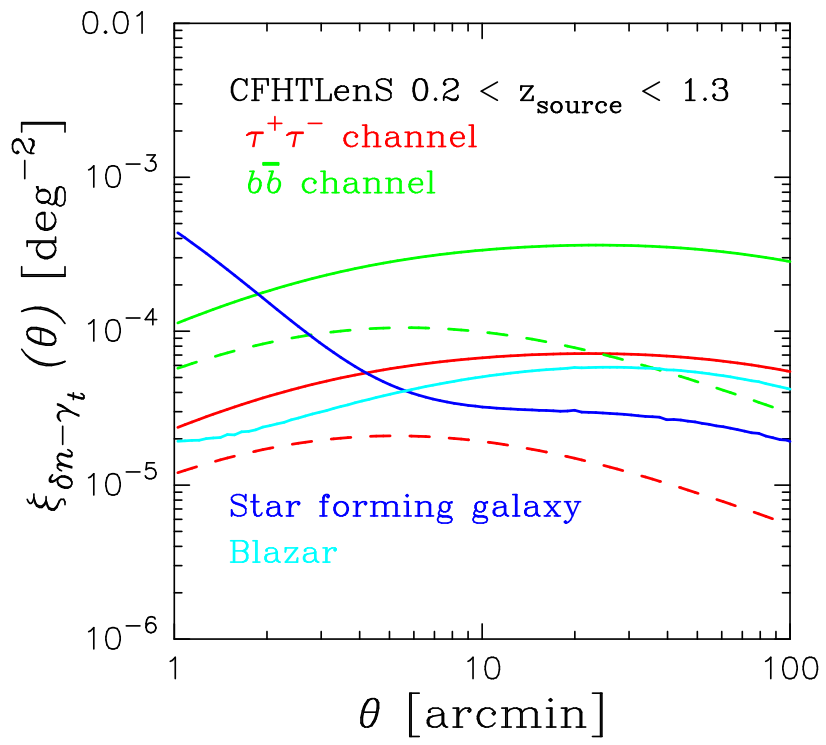

FIG. 3. The expected cross-correlation signals of cosmic shear and important components of the EGB: from SFG (blue), blazers (cyan), and DM annihilation. For the latter, we show the signal from a $100 \mathrm{GeV}$ DM particle with annihilation cross section $\langle\sigma v\rangle=3 \times 10^{-26} \mathrm{~cm}^{3} \mathrm{~s}^{-1}$ and annihilation channels $\tau^{+} \tau^{-}$(red) and $b \bar{b}$ (green). Furthermore we consider two values for the minimum halo mass; $M_{\min }=10^{-6} M_{\odot}($ solid $)$ and $M_{\min }=10^{6} M_{\odot}($ dashed $)$.

For the gamma-ray luminosity function of SFGs, we use the tight correlation between the infrared (IR) luminosity and the gamma-ray luminosity [13], and use the observed IR luminosity function [59]. We define gamma-ray luminosity in the energy range between 0.1 $\mathrm{GeV}$ and $100 \mathrm{GeV}$, and we assume a power-law spectrum with index $\alpha=2.7$ for SFGs. This leads to $N_{0}(\chi)=\left(\langle L\rangle / E_{0}^{2}\right)(\alpha-2) /(1+z(\chi))^{2-\alpha}$ so that the mean luminosity is obtained as $\langle L\rangle=\int \mathrm{d} E_{\gamma} E_{\gamma} N_{0}(\chi)\left(E_{\gamma} / E_{0}\right)^{-\alpha}$ with the integral performed from $(1+z) E_{0}$ to $(1+z) E_{1}$, where $E_{0}=100 \mathrm{MeV}$ and $E_{1}=100 \mathrm{GeV}$. We use the mass-luminosity relation for SFGs, $M(L)=10^{12} M_{\odot}\left(L / 10^{39} \mathrm{erg} \mathrm{s}^{-1}\right)^{0.5}$ that is calibrated by the Milky Way properties [21]. The minimum luminosity is set to $10^{30} \mathrm{erg} \mathrm{s}^{-1}$ at any redshift, while the maximum luminosity is estimated in the same way as in the case of blazars.

Figure 3 shows our benchmark model of cross-correlation signals in the case of DM annihilation with $m_{d m}=100 \mathrm{GeV}$ and $\langle\sigma v\rangle=3 \times 10^{-26} \mathrm{~cm}^{3} \mathrm{~s}^{-1}$. In this figure, the results for two annihilation channels are shown, the $\tau^{+} \tau^{-}$channel (red lines) and the $b \bar{b}$ channel (green lines). We show the level of model uncertainty due to the minimum halo mass $M_{\min }$ by 
plotting both the optimistic case with $M_{\min }=10^{-6} M_{\odot}$ (solid lines) and the conservative case with $M_{\min }=10^{6} M_{\odot}$ (dashed lines). The figure clearly shows the sensitivity of the results on $M_{\min }$ and the different annihilation channels. The blue and cyan line in figure 3 show the cross-correlation signals of cosmic shear and EGB contributed by unresolved SFGs and blazars, respectively. Clearly, the contribution from astrophysical sources can be significant at all angular scales. We note that our adopted model of blazars is different from the one in the previous work of Ref. [21]. Our model reproduces the observed flux counts of resolved blazars, whereas the model in Ref. [21] is aimed at reproducing the flux counts as well as the anisotropy of the EGB [11]. The main difference lies in the faint slope of the gamma-ray luminosity function. Overall, our model predicts a larger contribution from blazers to the EGB intensity than the model of Ref. [21] by a factor of $\sim 10$. The large model-difference unfortunately limits the extent to which we can subtract astrophysical contributions. In this paper, we first examine the case where DM annihilation is the sole contributor to the cross-correlation signal. Our analysis under this assumption should provide a conservative constraint on DM annihilation, because the astrophysical sources are expected to yield positive cross-correlation signals unless they are distributed in an anti-correlated manner with respect the underlying DM density field. Furthermore, we find that the statistical error in the current dataset is larger than the expected cross- correlation signals due to astrophysical sources. Therefore, the final result is not strongly dependent on the details of the models for the astrophysical sources.

\section{Point spread function}

The observed number of EGB photons along a line of sight $\boldsymbol{\theta}$ is expressed by the convolution of the underlying number of EGB photons with the PSF of the detector,

$$
\delta n^{\mathrm{obs}}(\boldsymbol{\theta})=\int \mathrm{d}^{2} \theta^{\prime} W_{\mathrm{PSF}}\left(\boldsymbol{\theta}-\boldsymbol{\theta}^{\prime}\right) \delta n\left(\boldsymbol{\theta}^{\prime}\right)
$$

where $\delta n^{\text {obs }}$ is the observed number of EGB photons and $W_{\mathrm{PSF}}$ is the PSF. This causes an additional scale dependence of the weight function of EGB counts in Eqs. (12) and (27). Considering the energy dependence of the PSF, the scale-dependent weight function is given by

$$
W_{g}(\chi) \rightarrow W_{g}(\chi, \ell)=\left.\int_{E_{\gamma, \min }}^{E_{\gamma, \max }} \mathrm{d} E_{\gamma} \frac{\langle\sigma v\rangle}{8 \pi}\left(\frac{\bar{\rho}_{\mathrm{dm}, 0}}{m_{\mathrm{dm}}}\right)^{2}[1+z(\chi)]^{3} \frac{\mathrm{d} N_{\gamma}}{\mathrm{d} E_{\gamma}}\right|_{E_{\gamma}^{\prime}}
$$




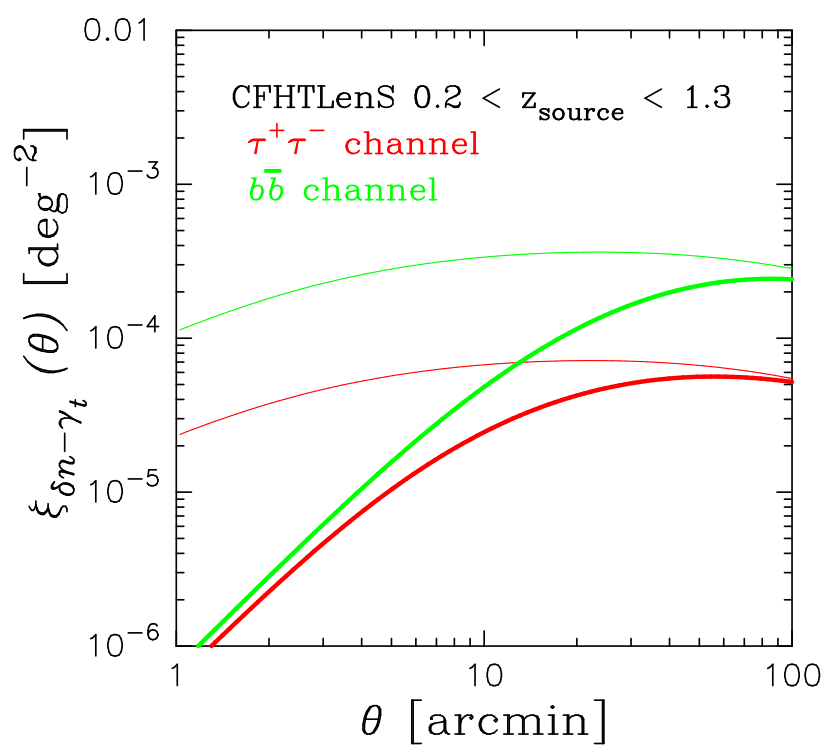

FIG. 4. The smoothing effect due to the PSF on the cross-correlation signals of cosmic shear and EGB. The thin lines represent the original expected signal as in Figure 3, annihilation of a 100 $\mathrm{GeV}$ mass DM with annihilation cross section $\langle\sigma v\rangle=3 \times 10^{-26} \mathrm{~cm}^{3} \mathrm{~s}^{-1}$ and minimum halo mass $M_{\text {min }}=10^{-6} M_{\odot} ;$ red and green lines are for the $\tau^{+} \tau^{-}$and $b \bar{b}$ channel, respectively, while the thick lines show the signal with smoothing due to the PSF.

$$
\begin{aligned}
& \times \exp \left[-\tau\left(E_{\gamma}^{\prime}, \chi\right)\right] \eta\left(E_{\gamma}\right) \tilde{W}_{\mathrm{PSF}}\left(\ell, E_{\gamma}\right), \\
W_{g, \text { ast }}(\chi) \rightarrow W_{g, \text { ast }}(\chi, \ell)=\int_{E_{\min }}^{E_{\max }} \frac{\mathrm{d} E_{\gamma}}{4 \pi} N_{0}(\chi)\left(\frac{E_{\gamma}^{\prime}}{E_{0}}\right)^{-\alpha} & \times \exp \left[-\tau\left(E_{\gamma}^{\prime}, \chi\right)\right] \eta\left(E_{\gamma}\right) \tilde{W}_{\mathrm{PSF}}\left(\ell, E_{\gamma}\right),
\end{aligned}
$$

where $\tilde{W}_{\mathrm{PSF}}\left(\ell, E_{\gamma}\right)$ is the fourier transform of the PSF.

In the case of Fermi-LAT, the PSF is modeled using the following functional form [60]:

$$
\begin{aligned}
W_{\mathrm{PSF}}\left(\theta, E_{\gamma}\right) & =A\left(E_{\gamma}\right)\left[f_{\text {core }} K\left(x, \sigma_{\text {core }}, \gamma_{\text {core }}\right)+\left(1-f_{\text {core }}\right) K\left(x, \sigma_{\text {tail }}, \gamma_{\text {tail }}\right)\right] \\
f_{\text {core }} & =\frac{1}{1+N_{\text {tail }} \sigma_{\text {tail }}^{2} / \sigma_{\text {core }}^{2}} \\
K(x, \sigma, \gamma) & =\frac{1}{2 \pi \sigma^{2}}\left(1-\frac{1}{\gamma}\right)\left[1+\frac{1}{2 \gamma} \frac{x^{2}}{\sigma^{2}}\right]^{-\gamma},
\end{aligned}
$$

where $x$ is a scaled-angular deviation defined by $x=\theta / S_{\mathrm{P}}\left(E_{\gamma}\right)$ and $A\left(E_{\gamma}\right)$ is the normalization factor such that $\int \mathrm{d}^{2} \theta W_{\mathrm{PSF}}\left(\theta, E_{\gamma}\right)=1$. The scale factor $S_{\mathrm{P}}\left(E_{\gamma}\right)$ is [60],

$$
S_{\mathrm{P}}\left(E_{\gamma}\right)=\sqrt{\left[c_{0}\left(\frac{E_{\gamma}}{100 \mathrm{MeV}}\right)^{-\beta}\right]^{2}+c_{1}^{2}},
$$


and the normalization is given by $A\left(E_{\gamma}\right)=\left[S_{\mathrm{P}}\left(E_{\gamma}\right)\right]^{2}$. In the present paper, we adopt the parameters estimated in the latest in-flight PSF for ULTRACLEAN photons 2 , i.e., $c_{0}=3.16$ $\operatorname{deg}$ and $c_{1}=0.034 \mathrm{deg}$ for front-converting events, and $c_{0}=5.32 \mathrm{deg}$ and $c_{1}=0.096 \mathrm{deg}$ for back-converting events, along with $\beta=0.8, N_{\text {tail }}=0.08639, \sigma_{\text {core }}=0.5399, \sigma_{\text {tail }}=1.063$, $\gamma_{\text {core }}=2.631$, and $\gamma_{\text {tail }}=2.932$ for both events [60].

Using the specific functional form shown in Eq. (33), we estimate the effect of the PSF on the cross-correlation analysis. In Figure 4, we consider the cross-correlation signal due to the annihilation of DM with $m_{\mathrm{dm}}=100 \mathrm{GeV}$ and $\langle\sigma v\rangle=3 \times 10^{-26} \mathrm{~cm}^{3} \mathrm{~s}^{-1}$. To account for the PSF, we first calculate the cross-correlation signals with the scale-dependent weight function in Eqs. (31) and (32) for front- and back-converting events, respectively. We then average these two signals at a given angular separation assuming the number of front-converting events is equal to that of back-converting events. Clearly, the smoothing effect significantly affects the cross-correlation signal especially at smaller angular scales than the typical size of the PSF, i.e. $\sim 50$ arcmin. We also expect that the pixelization effect would be unimportant in our analysis, because the pixel size is smaller than the size of the PSF (12 arcmin).

\section{RESULT}

We present the measurement of the cross-correlation signals of the cosmic shear and the EGB and discuss the implications. Figure 5 shows the cross-correlation signals obtained for each CFHTLenS patch. In each panel of Figure 5, we also show the cross-correlation using another component of weak lensing shear that is rotated $45^{\circ}$ from the tangential shear component. We refer to this component as $\gamma_{\times}$. In practice, $\gamma_{\times}$is often used as an indicator of systematics in the shape measurement. In the case of perfect shape measurement and no intrinsic alignment, the correlation signal with $\gamma_{\times}$should vanish statistically. In order to quantify the significance of the measured cross-correlation signals with respect to the statistical error, we use the $\chi^{2}$ statistics defined by

$$
\chi^{2}=\sum_{i, j} \xi_{\delta n-\gamma_{t}}\left(\theta_{i}\right) C_{i j}^{-1} \xi_{\delta n-\gamma_{t}}\left(\theta_{j}\right)
$$

where $\boldsymbol{C}^{-1}$ denotes the inverse covariance matrix estimated from the randomized realization shown in Section IVA. In our analysis, the number of deg of freedom is 10. The resulting

\footnotetext{
${ }^{2}$ made publicly available at
} http://fermi.gsfc.nasa.gov/ssc/data/analysis/documentation/Cicerone/Cicerone_LAT_IRFs/IRF_PSF.html 

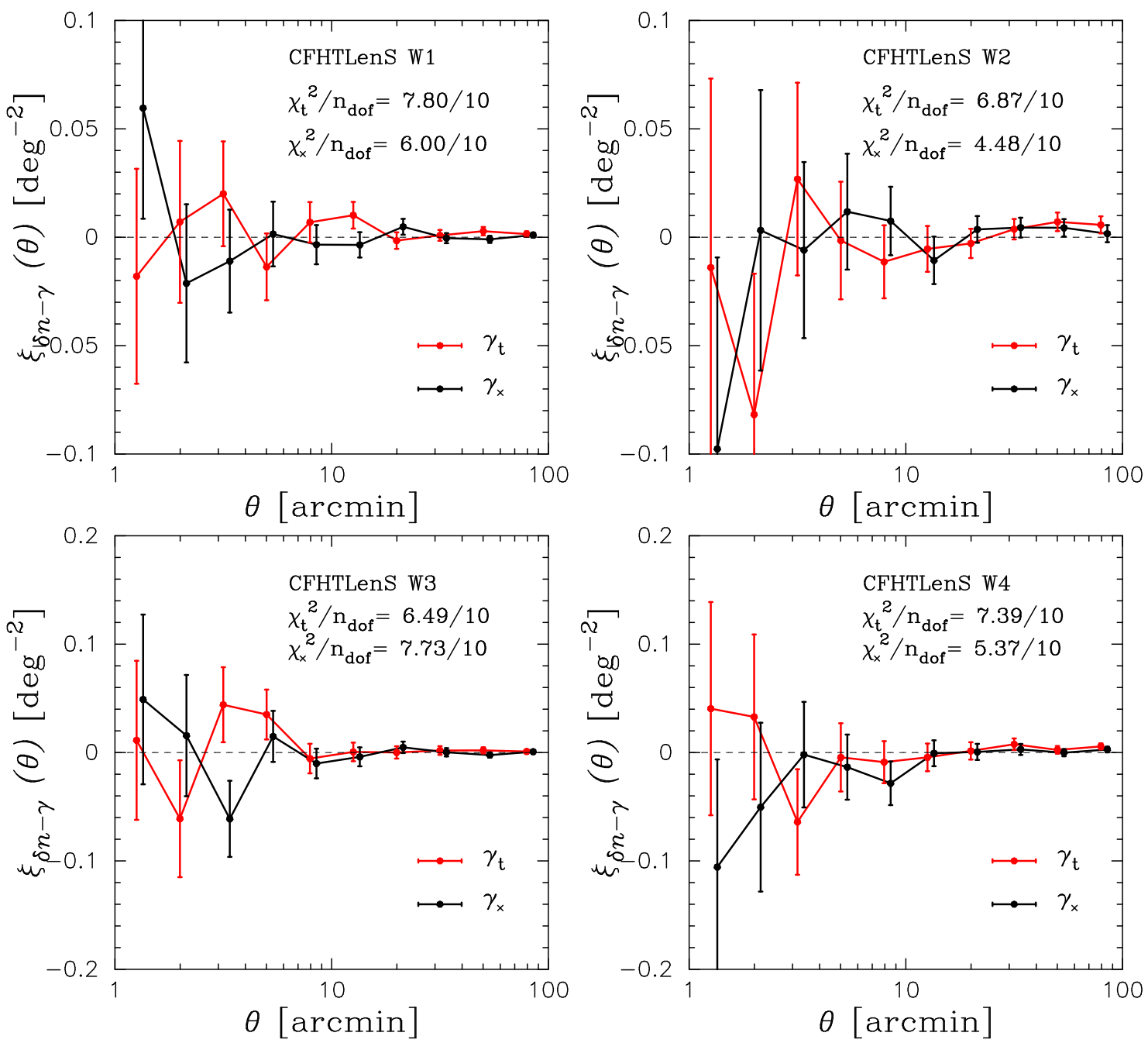

FIG. 5. The cross-correlation signal of cosmic shear and the EGB. Each panel corresponds to each of the CFHTLenS patches W1-W4. The red points show the result using tangential shear $\gamma_{+}$, while the black points are for $\gamma_{x}$. The error bars indicate the standard deviation estimated from our 500 randomized shear catalogues and 500 randomized photon count maps.

values of $\chi^{2} / n_{\text {dof }}$ for $\gamma_{t}$ and for $\gamma_{\times}$are shown in each panel. The result is consistent with null detection in each CFHTLenS patch. We confirm that the combined four field together is also consistent with null detection $\left(\chi^{2} / n_{\text {dof }}=[7.80+6.87+6.49+7.39] / 40=28.55 / 40\right.$ in total).

We are now able to use the null detection of the cross-correlation to place constraints on the DM annihilation cross-section. For this purpose, we use the maximum Likelihood analysis. We assume that the data vector $\boldsymbol{D}$ is well approximated by the multivariate 


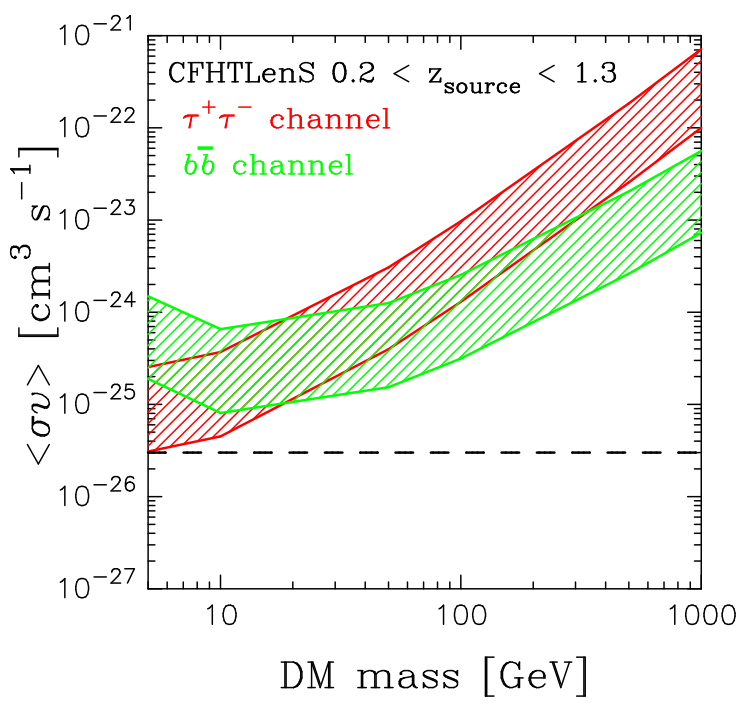

FIG. 6. The $68 \%$ confidence level upper limits on $\langle\sigma v\rangle$ as a function of DM mass. The red shaded region shows the upper bound for the $\tau^{+} \tau^{-}$channel and the green region is for the $b \bar{b}$ channel. Note that the widths of the shaded regions indicate the model uncertainty: for each shaded region, the upper curve is derived by our benchmark model with $M_{\min }=10^{6} M_{\odot}$ and the lower curve is obtained from the model with $M_{\min }=10^{-6} M_{\odot}$.

Gaussian distribution with covariance $\boldsymbol{C}$. In this case, $\chi^{2}$ statistics (log-likelihood) is given by

$$
\chi^{2}(\boldsymbol{p})=\sum_{i, j}\left(D_{i}-\mu_{i}(\boldsymbol{p})\right) C_{i j}^{-1}\left(D_{j}-\mu_{j}(\boldsymbol{p})\right),
$$

where $\boldsymbol{\mu}(\boldsymbol{p})$ is the theoretical prediction as a function of parameters of interest. In this paper, we use the halo model approach shown in Section IVB to calculate the theoretical prediction. For parameters of interest $\boldsymbol{p}$, we simply consider the DM particle mass and the annihilation cross-section, $m_{\mathrm{dm}}$ and $\langle\sigma v\rangle 3$ The data vector $\boldsymbol{D}$ consists of the measured cross-correlation signals with the range of $\theta=[1,100]$ arcmin as

$$
D_{i}=\left\{\xi_{\delta n-\gamma_{t}}\left(\theta_{1}\right), \xi_{\delta n-\gamma_{t}}\left(\theta_{2}\right), \ldots, \xi_{\delta n-\gamma_{t}}\left(\theta_{10}\right)\right\}
$$

where $\theta_{i}$ is the $i$-th bin of angular separation. The inverse covariance matrix $\boldsymbol{C}^{-1}$ includes the statistical error of the shape measurement and the photon Poisson error. In our likelihood

3 Strictly speaking, we need to consider other parameters associated with the model of substructure within DM haloes. These are, for example, the concentration parameter $c_{\mathrm{vir}}$ of host halo, subhalo density profile and subhalo mass function. Although we do not include these parameters explicitly in our analysis, we explore the overall effect by considering two cases with the different minimum halo mass $M_{\min }$ as the most important effective uncertainty of our benchanark model. 
analysis, we assume that the four CFHTLenS patches are independent of each other. With this assumption, the total log-likelihood is given by the summation of Eq. (38) in each CFHTLenS patch. In order to constrain $m_{\mathrm{dm}}$ and $\langle\sigma v\rangle$, we consider the $68 \%$ confidence level of posterior distribution function of parameters. This is given by the contour line in the two dimensional space $\left(m_{\mathrm{dm}}\right.$ and $\left.\langle\sigma v\rangle\right)$, which is defined as

$$
\Delta \chi^{2}(\boldsymbol{p})=\chi^{2}(\boldsymbol{p})-\chi^{2}(\boldsymbol{\mu}=0)=2.30
$$

As discussed in Section IVB, the choice of the minimum halo mass affects the theoretical predictions by a factor of about ten. We therefore derive constraints based on the optimistic case with $M_{\min }=10^{-6} M_{\odot}$ and on the conservative case with $M_{\min }=10^{6} M_{\odot}$.

Figure 6] shows the result of our likelihood analysis on the DM parameter space $m_{\mathrm{dm}}$ and $\langle\sigma v\rangle$. We plot the constraints for two representative particle physics model, the $\tau^{+} \tau^{-}$ channel and the $b \bar{b}$ channel. We also show the results for the two choices of $M_{\min }$. The constraint for the small $M_{\text {min }}$ is significantly stronger, as expected. At low DM mass, the annihilation cross-section is more severely constrained for the $\tau^{+} \tau^{-}$channel, because of its harder gamma-ray spectra that contribute photons at sensitive energies than for the $b \bar{b}$ channel of the same DM mass. For reference, the horizontal dashed line indicates the canonical cross section of $\langle\sigma v\rangle=3 \times 10^{-26} \mathrm{~cm}^{3} \mathrm{~s}^{-1}$ for a thermally produced DM.

\section{A. Future forecast}

Future weak lensing surveys are aimed at measuring cosmic shear over a wide area of more than a thousand square degs. Such observational programs include the Subaru Hyper Suprime-Cam (HSC) 1, the Dark Energy Survey (DES) 2, and the Large Synoptic Survey Telescope (LSST) 3. It is interesting to explore the discovery potential of the upcoming cosmology surveys in terms of the DM particle properties. In this section, we consider two of these wide surveys with an area coverage of $1400 \mathrm{deg}^{2}$ (HSC) and $20000 \mathrm{deg}^{2}$ (LSST), by simply scaling the covariance matrix by a factor of 154/1400 or 154/20000, respectively. Assuming the same number density and redshift distribution of source galaxies as in the CFHTLenS, the expected constraints can be scaled by the effective survey area. The result

\footnotetext{
${ }^{1}$ http://www.naoj.org/Projects/HSC/j_index.html

2 http://www.darkenergysurvey.org/

${ }^{3}$ http://www.lsst.org/lsst/
} 

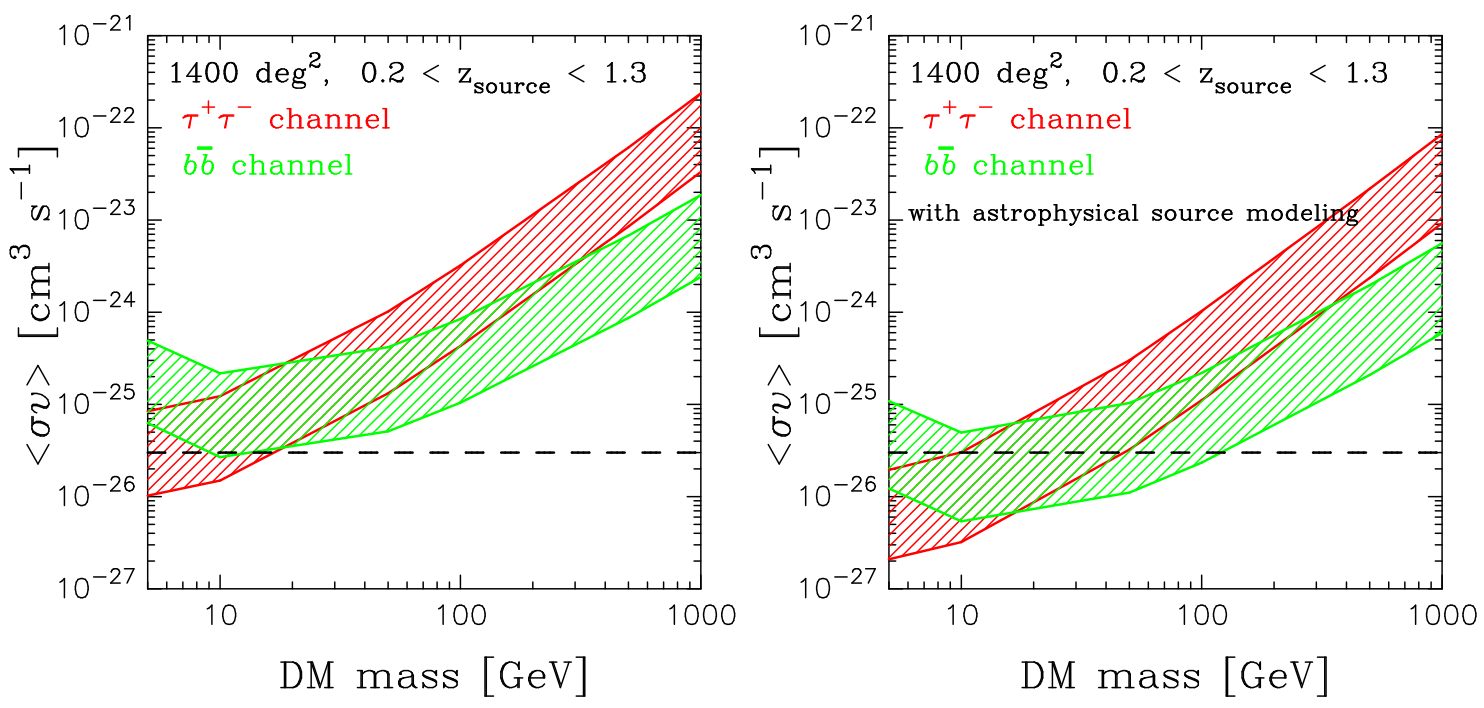

FIG. 7. We plot the expected $68 \%$ confidence level upper limit on $\langle\sigma v\rangle$ as a function of the DM mass for upcoming surveys. We show the case with a sky coverage of survey area $1400 \mathrm{deg}^{2}$. The red shaded region shows the expected upper limit for the $\tau^{+} \tau^{-}$channel and the green one for the $b \bar{b}$ channel. The left panel shows that the conservative case assuming the DM annihilation contribution only, while the right panel shows the optimistic case taking into account astrophysical sources.

suggests that the upper limit will be improved by a factor of $\sqrt{1400 / 154} \sim 3$ for HSC and by a factor of $\sqrt{20000 / 154} \sim 11$ for LSST. In particular, for a $100 \mathrm{GeV}$ DM, the upper limit of $\langle\sigma v\rangle$ with $68 \%$ confidence level could reach $2.7-22.2 \times 10^{-26} \mathrm{~cm}^{3} \mathrm{~s}^{-1}$ for $b \bar{b}$ channel and $1.1-8.51 \times 10^{-25} \mathrm{~cm}^{3} \mathrm{~s}^{-1}$ for $\tau^{+} \tau^{-}$channel in the case of the LSST-like survey. It will be important to include the uncertainty in the model template of galactic emission and also the sampling variance that is neglected in this paper. Then we will be able to derive robust and complementary probes of DM annihilation from the cross-correlation signal of cosmic shear and EGB.

As shown in Figure 3, the expected cross-correlation of astrophysical sources are comparable to the DM annihilation signal with $m_{\mathrm{dm}}=100 \mathrm{GeV}$ and $\langle\sigma v\rangle=3 \times 10^{-26} \mathrm{~cm}^{3} \mathrm{~s}^{-1}$. Thus it will be even more important to accurately take into account of the contribution of astrophysical sources such as blazars and SFG for future surveys. We thus include the contribution from the astrophysical sources on the assumption that the contribution of blazars and SFGs can be estimated as in our benchmark model described in IVB. The sum of the 


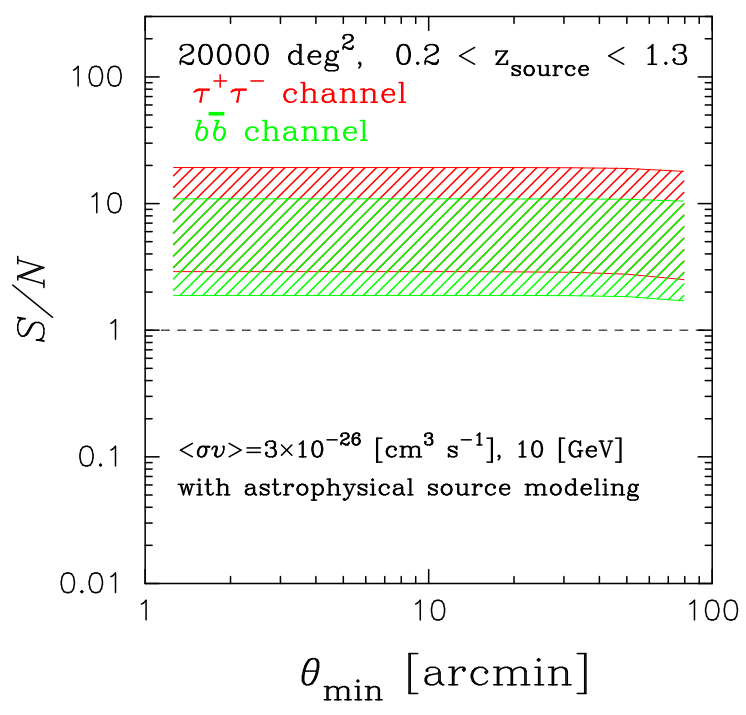

FIG. 8. The cumulative signal-to-noise ratio for the cross-correlation of cosmic shear and the EGB. We show the case with a sky coverage of survey area $20000 \mathrm{deg}^{2}$, i.e., a LSST like survey. The red shaded region shows the signal-to-noise ratio for the $\tau^{+} \tau^{-}$channel and the green one for the $b \bar{b}$ channel. We consider the sum of the DM annihilation contribution of a $10 \mathrm{GeV}$ mass DM and the astrophysical sources for these plots.

three contributions is given by

$$
\xi_{\delta n-\gamma_{t}}(\theta)=\xi_{\delta n-\gamma_{t}}^{\mathrm{dm}}\left(\theta \mid m_{\mathrm{dm}},\langle\sigma v\rangle\right)+\xi_{\delta n-\gamma_{t}}^{\mathrm{blazer}}(\theta)+\xi_{\delta n-\gamma_{t}}^{\mathrm{SFG}}(\theta)
$$

Using this as a theoretical model template, we perform the likelihood analysis to make forecast for DM constraints. For simplicity, we assume that the observed cross-correlation is identical to the one of the CFHTLenS W1 patch but that the covariance matrix can be scaled by the survey area. The expected constraint from the HSC-like survey is shown in Figure 7. The left panel shows the conservative case with no contribution from the astrophysical sources whereas the right panel shows the case with including the astrophysical sources. With the astrophysical sources in the model prediction, we can place tighter upper bound by $\sim 40-70 \%$ for the sky coverage of $1400 \mathrm{deg}^{2}$. It is clearly important to treat the contribution from the astrophysical sources carefully for future wide-field surveys.

We further study information content in the cross-correlation signal of cosmic shear and EGB. An important quantity is the cumulative signal-to-noise ratio $S / N$, which is defined 


$$
(S / N)^{2}=\sum_{i, j} \mu_{i}(\boldsymbol{p}) C_{i j}^{-1} \mu_{j}(\boldsymbol{p})
$$

In order to calculate $S / N$, we consider DM models with $\langle\sigma v\rangle=3 \times 10^{-26} \mathrm{~cm}^{3} \mathrm{~s}^{-1}$ for a 10 $\mathrm{GeV}$ and $100 \mathrm{GeV}$ dark matter and use the covariance matrix estimated by the randomized method shown in Section IVA.

Figure 8 shows the $S / N$ as a function of the minimum angular scale included in the cross-correlation analysis. In this figure, we consider the annihilation signal of a $10 \mathrm{GeV}$ DM particle and we set the maximum angular scale to 100 arcmin. Large-scale crosscorrelations determine the information content, and including data at small angular scales does not improve the significance. The same can be said of a $100 \mathrm{GeV}$ DM particle. This is simply because we can not extract information from cross-correlations on scales smaller than the size of the gamma-ray PSF. At large angular scales, $\theta \sim 100$ arcmin, the signals are mainly contributed by the DM annihilation. We expect that the cross-correlation analysis with upcoming survey with a large sky converge of $\sim 1000 \operatorname{deg}^{2}$ will be a powerful probe of dark matter annihilation. We also discuss the detectability of the cross-correlation signal with upcoming lensing surveys. In our benchmark model, the $S / N$ is almost proportional to $\langle\sigma v\rangle$ because the DM contribution dominates over astrophysical contributions. We can thus detect at a $3-\sigma$ confidence level the DM signature with $\langle\sigma v\rangle \simeq 3 \times 10^{-26} \mathrm{~cm}^{3} \mathrm{~s}^{-1}$ for a $10 \mathrm{GeV}$ dark matter and $\langle\sigma v\rangle \simeq 1 \times 10^{-25} \mathrm{~cm}^{3} \mathrm{~s}^{-1}$ for a $100 \mathrm{GeV}$ dark matter in a LSST-like survey. It is important to note that $S / N$ will likely increase significantly if cross-correlations at very large angular scales ( $\gtrsim 100$ arcmin) are included. In the present paper, the statistical error estimated from the real dataset is limited to the range of 1-100 arcmin. However, for upcoming wide-field surveys, we can measure the cross-correlation signal to much larger angular scales where the smoothing effect due to PSF is unimportant. To estimate the expected value of $S / N$ in upcoming surveys, one would need mock weak lensing catalogues and gamma-ray photon maps with a sky coverage of $\gtrsim 1000$ squared degs. This is along the line of our ongoing study using a large set of cosmological simulations in combination with actual Fermi all-sky observations. It is important to note that our method shown in the present paper probes the DM signature at cosmological scales, and thus is complementary to DM searches in local galaxies. 


\section{CONCLUSION AND DISCUSSION}

We have performed, for the first time, cross-correlation analysis of cosmic shear and the EGB using observational data from the CFHTLenS and the Fermi satellite. For the 154 square-degs sky coverage, the measured cross-correlation signal is consistent with null detection. Using theoretical models based on large-scale DM structure formation, we have estimated the statistical error from real data together with a large set of mock observations, and have placed constraints on the DM annihilation cross section. We have considered different DM annihilation channels and varied the minimum mass of DM halos. The derived constraint is $\langle\sigma v\rangle<10^{-25}-10^{-24} \mathrm{~cm}^{3} \mathrm{~s}^{-1}$ for a $100 \mathrm{GeV}$ DM, depending on the assumed parameters and annihilation channel. The constraint improves for smaller DM mass.

Recent analyses of the Fermi observations of dwarf galaxies [61 63] provide stronger constraints for DM annihilation. However, our constraints are derived using a completely different statistical method, based on the cross-correlation of the EGB and cosmic shear. The EGB intensity has been used to constrain the DM contribution, most recently by modeling and removing the astrophysical sources to obtain strong limits [64]. Our limits compete favorably with the constraints of Ref. [65] that use galaxy clusters and those of Ref. [43] that use anisotropies of the EGB. Given the range of potential DM signals in the literature and a broad range of potential particle candidates, complementary probes are critical to cast a wide net for DM signals and constraints. For example, recently a $\sim \mathrm{GeV}$ excess has been claimed towards the Galactic center whose spectral shape, normalization, and spatial morphology can all be explained by the annihilation of $10 \mathrm{GeV}(40 \mathrm{GeV})$ mass DM to $\tau^{+} \tau^{-}$ $(b \bar{b})$ with cross sections of $\langle\sigma v\rangle \sim 10^{-26} \mathrm{~cm}^{3} \mathrm{~s}^{-1}[66-73]$. The cross-correlation signal offers an independent method for testing the DM interpretation of the excess.

Encouraged by our initial study producing competitive constraints, we investigate the improvement expected with upcoming gravitational lensing survey with the sky coverage of 20000 square degs. We have shown that constrints on $\langle\sigma v\rangle$ would reach $2.7-22.2 \times$ $10^{-26} \mathrm{~cm}^{3} \mathrm{~s}^{-1}$ for the $b \bar{b}$ channel and $1.1-8.51 \times 10^{-25} \mathrm{~cm}^{3} \mathrm{~s}^{-1}$ for the $\tau^{+} \tau^{-}$channel, both for a $100 \mathrm{GeV}$ DM. For lighter DM motivated by the Galactic center excess, the constraints would reach $1.34-10.96 \times 10^{-26} \mathrm{~cm}^{3} \mathrm{~s}^{-1}$ for the $b \bar{b}$ channel (assuming $40 \mathrm{GeV}$ mass) and $0.39-3.24 \times 10^{-26} \mathrm{~cm}^{3} \mathrm{~s}^{-1}$ for the $\tau^{+} \tau^{-}$(assuming $10 \mathrm{GeV}$ mass), allowing a test of the DM origin of the Galactic center excess. Furthermore, if the accurate modeling of 


\begin{tabular}{|c|c|c|c|c|}
\hline & ev2/P7V6 & ev2/P7rep & ev4/P7V6 & ev4/P7rep \\
\hline W1 & $6.91 / 10$ & $6.22 / 10$ & $8.58 / 10$ & $7.80 / 10$ \\
\hline W2 & $12.26 / 10$ & $12.32 / 10$ & $6.98 / 10$ & $6.87 / 10$ \\
\hline W3 & $7.62 / 10$ & $7.11 / 10$ & $8.77 / 10$ & $6.49 / 10$ \\
\hline W4 & $12.88 / 10$ & $12.95 / 10$ & $7.57 / 10$ & $7.39 / 10$ \\
\hline
\end{tabular}

TABLE I. The impact of Fermi Galactic diffuse model on the cross-correlation analysis. We summarize the $\chi^{2}$ value of the cross-correlation signal in each CFHTLenS patch using different models and photon selections.

astrophysical contributions to the cross-correlation can be made, one can reasonably expect constraints on $\langle\sigma v\rangle$ to improve by $40-70 \%$ for a broad range of DM mass. Gamma-ray data also stand to improve. In this study we have used a conservative mask of $2^{\circ}$ around each point-source. While more aggressive masks or point-source modeling will increase photon statistics, these must be weighed by their larger systematic uncertainties. Also, at present, when we adopt a smaller mask of $1^{\circ}$ radius around each point source, we find that the errors on $\xi$ improved by only 10\%. Nevertheless, with more data, aggressive masks will become feasible. In particular, analyses that focus on higher energy photons, which due to their higher angular and energy resolutions can tolerate more aggressive masks, may yield improved probes especially at high DM masses.

Overall, these results suggest that the cross-correlation analysis of cosmic shear and the EGB will play a crucial role for the search for DM annihilation signatures. It is thus important to address a few issues in the cross-correlation analysis of cosmic shear and the EGB. First, in this paper, we have only implemented a crude estimate of the systematic error associated with the gamma-ray foreground subtraction. Second, we have not included the sampling variance. While these are not expected to be a significant source of uncertainties at present, mainly because of the large statistical error in the current data sets, they would become more important for analyses using data from upcoming surveys. For the diffuse model subtraction, we have made an attempt to estimate the systematics by employing different gamma-ray datasets and different Galactic diffuse emission models. The resulting

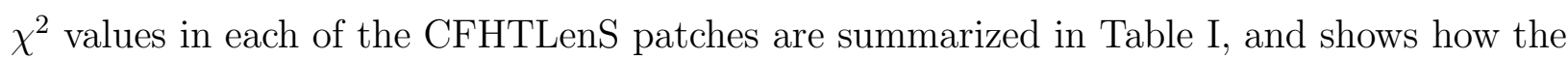
typical systematic error associated with Fermi photon analysis are very small $\left(\Delta \chi^{2} \sim 1-5\right)$. 
In the case of a LSST-like survey (see Section VA), this difference could induce a systematic error of $\langle\sigma v\rangle$ for a $100 \mathrm{GeV} \mathrm{DM}$ on the level of $\sim 3 \times 10^{-26} \mathrm{~cm}^{3} \mathrm{~s}^{-1}$ for both the $b \bar{b}$ channel and the $\tau^{+} \tau^{-}$channel.

Detailed comparisons with numerical simulations would also be needed to test the accuracy of our benchmark model based on halo model approach (see also Appendix B). Combined with other observabations such as the mean intensity of the EGB, angular cor-

relation of the EGB and the cross-correlation of galaxy position and the EGB [74], one can expect that some of the degeneracies between the DM annihilation and astrophysical sources may be broken. It is therefore important to investigate how much information of the EGB can be extracted from such combined analyses using multiple astrophysical datasets. Gamma-ray analyses with future cosmological surveys would be very powerful methods for understanding the origin of the EGB and the indirect search of DM annihilation.

\section{ACKNOWLEDGMENTS}

The authors are grateful to Kazuhiro Nakazawa for helpful discussion on cross correlation analysis. MS is supported by Research Fellowships of the Japan Society for the Promotion of Science (JSPS) for Young Scientists, and SH is supported by a Research Fellowship for Research Abroad by JSPS. NY acknowledges financial support from the JSPS Grant-inAid for Scientific Research (25287050). Numerical computations presented in this paper were in part carried out on the general-purpose PC farm at Center for Computational Astrophysics, CfCA, of National Astronomical Observatory of Japan. This work is based on observations obtained with MegaPrime/MegaCam, a joint project of CFHT and CEA/IRFU, at the Canada-France-Hawaii Telescope (CFHT) which is operated by the National Research Council (NRC) of Canada, the Institut National des Sciences de l'Univers of the Centre National de la Recherche Scientifique (CNRS) of France, and the University of Hawaii. The research used the facilities of the Canadian Astronomy Data Centre operated by the National Research Council of Canada with the support of the Canadian Space Agency. CFHTLenS data processing was made possible thanks to significant computing support from the NSERC Research Tools and Instruments grant program. 


\section{Appendix A: Covariance of Cross-Correlation Estimator}

Here, we summarize the properties of the estimator for cross-correlation analysis used in the present paper. Our estimator is given by Eq. (6). Let us consider a simple case in this appendix. When one measures galaxies' ellipticities $(\boldsymbol{\epsilon})$ and counts extragalactic gamma-ray photons $(\delta n)$ from an observed data set precisely, the cross-correlation estimator is expressed by

$$
\begin{aligned}
\hat{\xi}_{\delta n-\gamma_{t}}(\theta) & =\frac{1}{N_{\mathrm{p}}(\theta)} \sum_{i}^{N_{\text {pixel }}} \sum_{j}^{N_{\text {gal }}} \delta n\left(\boldsymbol{\phi}_{i}\right) \epsilon_{t}\left(\boldsymbol{\phi}_{j} \mid \boldsymbol{\phi}_{i}\right) \Delta_{\theta}\left(\boldsymbol{\phi}_{i}-\boldsymbol{\phi}_{j}\right), \\
N_{\mathrm{p}}(\theta) & =\sum_{i}^{N_{\text {pixel }}} \sum_{j}^{N_{\text {gal }}} \Delta_{\theta}\left(\boldsymbol{\phi}_{i}-\boldsymbol{\phi}_{j}\right),
\end{aligned}
$$

where $\Delta_{\theta}(\phi)=1$ for $\theta-\Delta \theta / 2 \leq \phi \leq \theta+\Delta \theta / 2$ and zero otherwise and $N_{\mathrm{p}}(\theta)$ represents the effective pair number in cross-correlation analysis. One can clearly see that this estimator is an unbiased estimator of of cross-correlation signal $\xi_{\delta n-\gamma_{t}}(\theta)$.

In order to discuss statistical significances of the measured estimator from real data, we need to estimate the covariance of $\hat{\xi}_{\delta n-\gamma_{t}}(\theta)$. In particular, the covariance in the case of $\left\langle\hat{\xi}_{\delta n-\gamma_{t}}(\theta)\right\rangle=0$ is needed for detection of cross-correlation signals. The covariance matrix of Eq. (A1) is defined by

$$
\begin{aligned}
\operatorname{Cov}\left[\hat{\xi}_{\delta n-\gamma_{t}}\left(\theta_{1}\right), \hat{\xi}_{\delta n-\gamma_{t}}\left(\theta_{2}\right)\right]= & \left\langle\left(\hat{\xi}_{\delta n-\gamma_{t}}\left(\theta_{1}\right)-\xi_{\delta n-\gamma_{t}}\left(\theta_{1}\right)\right)\left(\hat{\xi}_{\delta n-\gamma_{t}}\left(\theta_{2}\right)-\xi_{\delta n-\gamma_{t}}\left(\theta_{2}\right)\right)\right\rangle \\
= & \frac{1}{N_{\mathrm{p}}\left(\theta_{1}\right) N_{\mathrm{p}}\left(\theta_{2}\right)}\left[\sum_{i, j, k, \ell}\left\langle n\left(\boldsymbol{\phi}_{i}\right) \epsilon_{t}\left(\boldsymbol{\phi}_{j} \mid \boldsymbol{\phi}_{i}\right) n\left(\boldsymbol{\phi}_{k}\right) \epsilon_{t}\left(\boldsymbol{\phi}_{\ell} \mid \boldsymbol{\phi}_{k}\right)\right\rangle\right. \\
& \left.\times \Delta_{\theta_{1}}\left(\boldsymbol{\phi}_{i}-\boldsymbol{\phi}_{j}\right) \Delta_{\theta_{2}}\left(\boldsymbol{\phi}_{k}-\boldsymbol{\phi}_{\ell}\right)\right]-\xi_{\delta n-\gamma_{t}}\left(\theta_{1}\right) \xi_{\delta n-\gamma_{t}}\left(\theta_{2}\right),(\mathrm{A} 3)
\end{aligned}
$$

where $i$ and $k$ represents the indeces of summation over gamma-ray counts, and $j$ and $\ell$ are for galaxies. When two fields $\delta n$ and $\boldsymbol{\epsilon}$ are independent of each other, the ensemble average $\left\langle\delta n \epsilon_{t} \delta n \epsilon_{t}\right\rangle$ would simply reduce the ensemble average of each field, i.e. $\langle\delta n \delta n\rangle\left\langle\epsilon_{t} \epsilon_{t}\right\rangle$. For shape of galaxies, the two point correlation function $\left\langle\epsilon_{t} \epsilon_{t}\right\rangle$ would be expressed by the summation of intrinsic variance and the correlation signal due to large scale structure;

$$
\left\langle\epsilon_{t}\left(\boldsymbol{\phi}_{j}\right) \epsilon_{t}\left(\boldsymbol{\phi}_{\ell}\right)\right\rangle=\frac{\sigma_{\mathrm{int}}^{2}}{2} \delta_{j \ell}+\xi_{+}\left(\left|\boldsymbol{\phi}_{j}-\boldsymbol{\phi}_{\ell}\right|\right)
$$

where $\sigma_{\text {int }}$ represents the variance of intrinsic shape of galaxies and $\xi_{+}(\theta)$ is the two point correlation signal due to weak gravitational lensing. In a concordance $\Lambda$ CDM universe, $\xi_{+}(\theta)$ 
would be expected to be on the order of $10^{-4}$. The latest cosmic shear measurement [19] confirmed this expectation with high significance and shows that the typical value of $\sigma_{\text {int }}$ to be $\sim 0.4$. For extragalactic gamma-ray counts, the origin is still unknown. Hence, it is difficult to estimate the exact contribution to the two point correlation function $\langle\delta n \delta n\rangle$. At least, we expect that Poisson processes would dominate on scales larger than the PDF in gamma-ray surveys. We assume that photon count fluctuations follow a Poisson distribution with mean corresponding to $\delta n^{\text {obs }}(\phi)$, where $\delta n^{\text {obs }}(\phi)$ is the observed gamma-ray count map. In this case, two point correlation function $\langle\delta n \delta n\rangle$ would be expressed by

$$
\left\langle\delta n\left(\boldsymbol{\phi}_{i}\right) \delta n\left(\boldsymbol{\phi}_{k}\right)\right\rangle=\delta n^{\mathrm{obs}}\left(\boldsymbol{\phi}_{i}\right) \delta_{i k}+\delta n^{\mathrm{obs}}\left(\boldsymbol{\phi}_{i}\right) \delta n^{\mathrm{obs}}\left(\boldsymbol{\phi}_{k}\right),
$$

where the first term represents Poisson fluctuations in count maps and the second term includes the effect of correlation due to the point spread function in gamma-ray surveys. Eq. (A5) would be a reasonable approximation when considering scales larger than the size of point spread function, i.e. $\sim 1 \mathrm{deg}$ in our analysis.

Using Eqs. (A4) and (A5), and $\left\langle\hat{\xi}_{\delta n-\gamma_{t}}(\theta)\right\rangle=0$, we can divide the covariance of our estimator into four contributions as follows:

$$
\begin{aligned}
& \operatorname{Cov}\left[\hat{\xi}_{\delta n-\gamma_{t}}\left(\theta_{1}\right), \hat{\xi}_{\delta n-\gamma_{t}}\left(\theta_{2}\right)\right]=C_{\mathrm{SN}+\mathrm{p}}\left(\theta_{1}, \theta_{2}\right)+C_{\mathrm{WL}+\mathrm{p}}\left(\theta_{1}, \theta_{2}\right) \\
& C_{\mathrm{SN}+\mathrm{p}}\left(\theta_{1}, \theta_{2}\right)=\frac{1}{N_{\mathrm{p}}\left(\theta_{1}\right) N_{\mathrm{p}}\left(\theta_{2}\right)} \sum_{i, j} \delta n^{\mathrm{obs}}\left(\boldsymbol{\phi}_{i}\right) \frac{\sigma_{\mathrm{int}}^{2}}{2} \Delta_{\theta_{1}}(i j) \Delta_{\theta_{2}}(i j), \\
& C_{\mathrm{WL}+\mathrm{p}}\left(\theta_{1}, \theta_{2}\right)=\frac{1}{N_{\mathrm{p}}\left(\theta_{1}\right) N_{\mathrm{p}}\left(\theta_{2}\right)} \sum_{i, j, \ell} \delta n^{\mathrm{obs}}\left(\boldsymbol{\phi}_{i}\right) \xi_{+}\left(\left|\boldsymbol{\phi}_{j}-\boldsymbol{\phi}_{\ell}\right|\right) \Delta_{\theta_{1}}(i j) \Delta_{\theta_{2}}(i \ell),(\mathrm{A} 8 \\
& C_{\mathrm{SN}+\mathrm{obs}}\left(\theta_{1}, \theta_{2}\right)=\frac{1}{N_{\mathrm{p}}\left(\theta_{1}\right) N_{\mathrm{p}}\left(\theta_{2}\right)} \sum_{i, j, k} \delta n^{\mathrm{obs}}\left(\boldsymbol{\phi}_{i}\right) \delta n^{\mathrm{obs}}\left(\boldsymbol{\phi}_{k}\right) \frac{\sigma_{\text {int }}^{2}}{2} \Delta_{\theta_{1}}(i j) \Delta_{\theta_{2}}(k j)(, \mathrm{A}) \\
& C_{\mathrm{WL}+\mathrm{obs}}\left(\theta_{1}, \theta_{2}\right)=\frac{1}{N_{\mathrm{p}}\left(\theta_{1}\right) N_{\mathrm{p}}\left(\theta_{2}\right)}\left[\sum_{i, j, k, \ell} \delta n^{\mathrm{obs}}\left(\boldsymbol{\phi}_{i}\right) n^{\mathrm{obs}}\left(\boldsymbol{\phi}_{k}\right) \xi_{+}\left(\left|\boldsymbol{\phi}_{j}-\boldsymbol{\phi}_{\ell}\right|\right)\right. \\
&\left.\times \Delta_{\theta_{1}}(i j) \Delta_{\theta_{2}}(k \ell)\right]
\end{aligned}
$$

where $\Delta_{\theta_{1}}(i j)=\Delta_{\theta_{1}}\left(\phi_{i}-\phi_{j}\right)$ and so on. According to the observational fact that $\xi_{+}$is smaller than $\sigma_{\text {int }}^{2}$ by a factor of $10^{-3}$, the dominant contributions in Eq. (A6) would be the first term $C_{\mathrm{SN}+\mathrm{p}}$ and the third term $C_{\mathrm{SN}+\mathrm{obs}} \cdot C_{\mathrm{SN}+\mathrm{p}}$ is estimated from the observed galaxy catalogue and random count maps based on Poisson distribution. We can also estimate $C_{\mathrm{SN}+\text { obs }}$ by cross-correlating the observed photon counts and randomized galaxy catalogues. The estimation of $C_{\mathrm{SN}+\mathrm{p}}$ and $C_{\mathrm{SN}+\mathrm{obs}}$ from the real data set is found in Section IV A. 


\section{Appendix B: Effect Of Dark Matter Halo Profile Uncertainties On Cross-Correlation}

Signals

Here, we quantity the effect of uncertainties of the DM halo profiles on the cross- correlation between cosmic shear and the EGB. In order to calculate the theoretical model of cross-correlation signals, we follow the halo model approach as in Section IVB. The halo model posits that there are mainly two contributions of the cross-correlation signal: the one-halo term and the two-halo term. For a given length scale $k$, the main contribution to the one-halo term as calculated by Eq. (22) comes from galaxy cluster size halos with $10^{13}-10^{15} M_{\odot}$. This is valid for the two-halo term associated with density fluctuations (i.e., the first integral in Eq. (23) $)$. On the other hand, the two-halo term associated with density squared (i.e., the second integral in Eq. (23)) is mainly determined by the smoothed profile contribution $\int \mathrm{d} V \rho_{h}^{2}(r \mid M, z)$ with dominant contribution from lower mass scales. Assuming that the concentration parameter $c_{\text {vir }}=r_{\text {vir }} / r_{s} \propto M^{\alpha}$ with $\alpha \sim-0.1$, $M n(M, z) \int \mathrm{d} V \rho_{h}^{2}(r \mid M, z)$ would scaled as $\sim M^{3 \alpha}$ for $M<10^{12} M_{\odot}$. This fact implies that the low mass halos dominates the two-halo term and that the overall amplitude of the twohalo term is sensitive to the minimum halo mass. Thus, along with $M_{\min }, c_{\mathrm{vir}}(z, M)$ is one of the most important parameters in the halo model.

Recent numerical simulations [e.g., 75] suggest a non-monotonic relation between the concentration parameter and the mass of DM haloes. In this appendix, we test the dependence of the cross-correlation signal on $c_{\mathrm{vir}}(z, M)$ by comparing a simple power-law model and the non-monotonic model. For the non-monotonic $c_{\mathrm{vir}}(z, M)$ model, we use the fitting function of Ref. [75] that determines $c_{\mathrm{vir}}$ as a function of the linear $r m s$ density fluctuation $\sigma(z, M)$. This fitting function successfully reproduces the complex feature of $c_{\text {vir }}$ found in numerical simulations. For the power-law model, we apply the functional form shown in Ref. [55] as in our benchmark model.

Figure 9 shows the comparison between the halo model calculations with the power-law and non-monotonic models of $c_{\text {vir }}$. Each solid line is the same as the one shown in figure 3 , The dashed-dotted lines correspond to the halo model with the non-monotonic model of $c_{\text {vir }}$. For the non-monotonic model of $c_{\mathrm{vir}}$, we found that the final result is much less sensitive to the minimum halo mass because of the flattening feature of $c_{\mathrm{vir}}$ at low masses. The most important result is perhaps that the cross-correlation signals would be dominated by the one- 


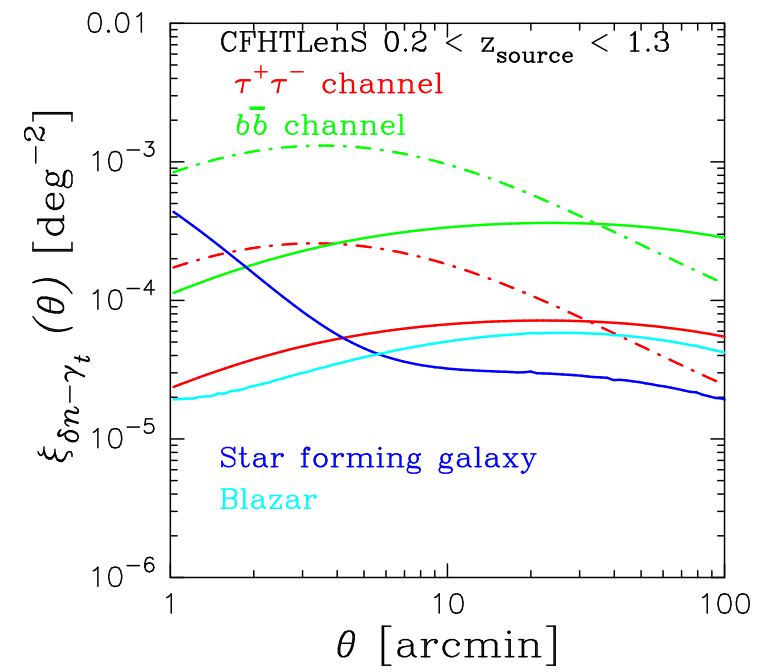

FIG. 9. The expected cross-correlation signals of cosmic shear and EGB from various sources. The signal from the annihilation of a $100 \mathrm{GeV}$ mass DM particle with annihilation cross section $\langle\sigma v\rangle=3 \times 10^{-26} \mathrm{~cm}^{3} \mathrm{~s}^{-1}$ is shown separately for the $\tau^{+} \tau^{-}$channel (red lines) and the $b \bar{b}$ channel (green lines). The solid lines shows the halo model with the power-low model of $c_{\text {vir }}$ with assumed minimum DM halo mass $M_{\min }=10^{-6} M_{\odot}$. The dashed-dotted line corresponds to the halo model calculation with the non-monotonic model of $c_{\mathrm{vir}}$. The blue and cyan line show the contribution from SFG and blazars, respectively.

halo term for the non-monotonic model, which is different from the result of our benchmark model and from previous work [21]. This is mainly due to the higher concentration in massive DM haloes than in our benchmark model. Consequently, the expected signals for the non-monotonic model would be ten times as large as our benchmark model for smaller angular scale at $\theta<10$ arcmin. However, for the angular scale larger than 30 arcmin, the two models with the different $c_{\text {vir }}$ show quite similar amplitudes of the cross-correlation. Clearly, the choice of $c_{\text {vir }}$ model would not affect the final constraints of DM annihilation significantly because most of the information about DM annihilation come from the large scale clustering as shown in Section VA. Figure 10 shows the $68 \%$ confidence upper limit of DM annihilation obtained from the current data set shown in Section III with the nonmonotonic model of $c_{\text {vir. }}$. In figure 10, we simply assume that DM annihilation is the only contribution to the cross-correlation signals and take into account the smoothing effect due to PSF in the same manner shown in Section IVB. We found the constraints on $\langle\sigma v\rangle$ degrade 


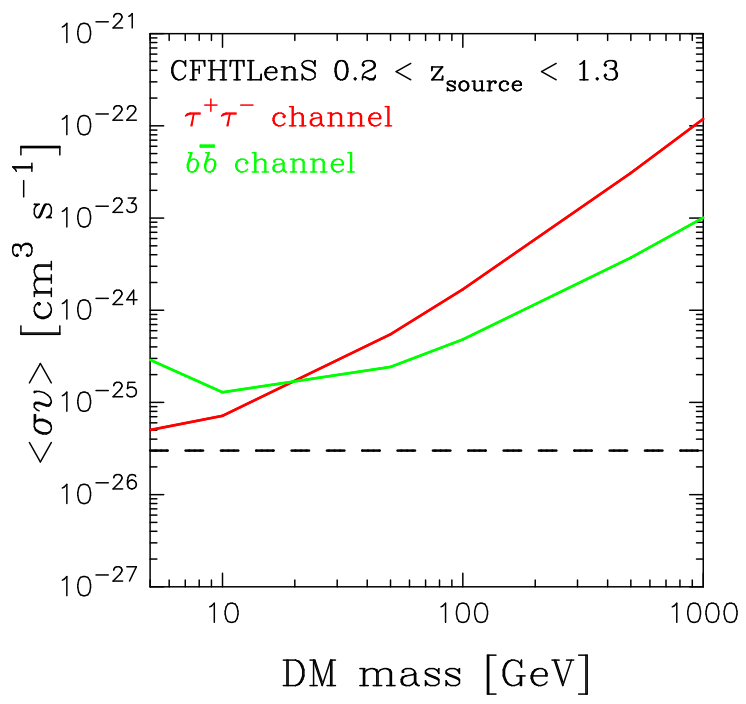

FIG. 10. The $68 \%$ confidence level upper limits on $\langle\sigma v\rangle$ as functions of the DM mass. The red shaded region shows the upper limit for the $\tau^{+} \tau^{-}$channel and the green one for the $b \bar{b}$ channel.

by $\sim 10 \%$ over a wide mass range of $5-1000 \mathrm{GeV}$.

[1] W. L. Kraushaar, G. W. Clark, G. P. Garmire, R. Borken, P. Higbie, V. Leong, and T. Thorsos, Astrophys.J. 177, 341 (1972).

[2] C. E. Fichtel, D. A. Kniffen, and R. C. Hartman, Astrophys.J.Lett. 186, L99 (1973).

[3] P. Sreekumar et al. (EGRET Collaboration), Astrophys.J. 494,523 (1998), arXiv:astro-ph/9709257 [astro-ph].

[4] A. Abdo et al. (Fermi-LAT collaboration), Phys.Rev.Lett. 104, 101101 (2010), arXiv:1002.3603 [astro-ph.HE],

[5] F. Stecker and M. Salamon, Astrophys.J. 464, 600 (1996), arXiv:astro-ph/9601120 [astro-ph].

[6] T. Narumoto and T. Totani, Astrophys.J. 643, 81 (2006), arXiv:astro-ph/0602178 [astro-ph].

[7] Y. Inoue and T. Totani, Astrophys.J. 702, 523 (2009), arXiv:0810.3580 [astro-ph].

[8] M. Ackermann et al. (Fermi LAT Collaboration), Phys.Rev. D85, 083007 (2012), arXiv:1202.2856 [astro-ph.HE].

[9] A. Abdo et al. (Fermi-LAT Collaboration), Astrophys.J. 720, 435 (2010), arXiv:1003.0895 [astro-ph.CO]. 
[10] M. Ajello, M. Shaw, R. Romani, C. Dermer, L. Costamante, et al., Astrophys.J. 751, 108 (2012), arXiv:1110.3787 [astro-ph.CO],

[11] J. P. Harding and K. N. Abazajian, JCAP 1211, 026 (2012), arXiv:1206.4734 [astro-ph.HE].

[12] A. Cuoco, E. Komatsu, and J. Siegal-Gaskins, Phys.Rev. D86, 063004 (2012), arXiv:1202.5309 [astro-ph.CO].

[13] M. Ackermann et al. (Fermi LAT Collaboration), Astrophys.J. 755, 164 (2012), arXiv:1206.1346 [astro-ph.HE],

[14] Y. Inoue, Astrophys.J. 733, 66 (2011), arXiv:1103.3946 [astro-ph.HE].

[15] E. Komatsu et al. (WMAP Collaboration), Astrophys.J.Suppl. 192, 18 (2011) arXiv:1001.4538 [astro-ph.CO],

[16] P. Ade et al. (Planck Collaboration), Astron.Astrophys. (2014), 10.1051/0004-6361/201321591. arXiv:1303.5076 [astro-ph.CO].

[17] M. Tegmark et al. (SDSS Collaboration), Phys.Rev. D74, 123507 (2006) arXiv:astro-ph/0608632 [astro-ph].

[18] L. Anderson, E. Aubourg, S. Bailey, D. Bizyaev, M. Blanton, et al., Mon.Not.Roy.Astron.Soc. 427, 3435 (2012), arXiv:1203.6594 [astro-ph.CO],

[19] M. Kilbinger, L. Fu, C. Heymans, F. Simpson, J. Benjamin, et al., Mon.Not.Roy.Astron.Soc. 430, 2200 (2013), arXiv:1212.3338 [astro-ph.CO].

[20] S. Ando and E. Komatsu, Phys.Rev. D73, 023521 (2006), arXiv:astro-ph/0512217 [astro-ph],

[21] S. Camera, M. Fornasa, N. Fornengo, and M. Regis, Astrophys.J. 771, L5 (2013), arXiv:1212.5018 [astro-ph.CO],

[22] R. Gilmore, R. Somerville, J. Primack, and A. Dominguez, Mon.Not.Roy.Astron.Soc. 422, 3189 (2012), arXiv:1104.0671 [astro-ph.CO].

[23] M. Cirelli, G. Corcella, A. Hektor, G. Hutsi, M. Kadastik, et al., JCAP 1103, 051 (2011), arXiv:1012.4515 [hep-ph].

[24] M. Cirelli, P. D. Serpico, and G. Zaharijas, JCAP 1311, 035 (2013), arXiv:1307.7152 [astro-ph.HE],

[25] L. Bergstrom, T. Bringmann, M. Eriksson, and M. Gustafsson, Phys.Rev.Lett. 95, 241301 (2005), arXiv:hep-ph/0507229 [hep-ph].

[26] P.-J. Zhang and J. F. Beacom, Astrophys.J. 614, 37 (2004), arXiv:astro-ph/0401351 [astro-ph]. 
[27] S. S. Campbell and J. F. Beacom, (2013), arXiv:1312.3945 [astro-ph.HE].

[28] Y. Rasera, R. Teyssier, P. Sizun, B. Cordier, J. Paul, et al., Phys.Rev. D73, 103518 (2006), arXiv:astro-ph/0507707 [astro-ph].

[29] S. Hofmann, D. J. Schwarz, and H. Stoecker, Phys.Rev. D64, 083507 (2001), arXiv:astro-ph/0104173 [astro-ph].

[30] A. Loeb and M. Zaldarriaga, Phys.Rev. D71, 103520 (2005), arXiv:astro-ph/0504112 [astro-ph].

[31] T. Bringmann, New J.Phys. 11, 105027 (2009), arXiv:0903.0189 [astro-ph.CO],

[32] T. Goerdt, O. Y. Gnedin, B. Moore, J. Diemand, and J. Stadel, Mon.Not.Roy.Astron.Soc. 375, 191 (2007), arXiv:astro-ph/0608495 [astro-ph].

[33] V. Berezinsky, V. Dokuchaev, and Y. Eroshenko, Phys.Rev. D77, 083519 (2008), arXiv:0712.3499 [astro-ph].

[34] C. Heymans, L. Van Waerbeke, L. Miller, T. Erben, H. Hildebrandt, et al., Mon.Not.Roy.Astron.Soc. 427, 146 (2012), arXiv:1210.0032 [astro-ph.CO].

[35] H. Hildebrandt, T. Erben, K. Kuijken, L. van Waerbeke, C. Heymans, et al., Mon.Not.Roy.Astron.Soc. 421, 2355 (2012), arXiv:1111.4434 [astro-ph.CO],

[36] T. Erben, H. Hildebrandt, L. Miller, L. van Waerbeke, C. Heymans, et al., Mon.Not.Roy.Astron.Soc. 433, 2545 (2013), arXiv:1210.8156 [astro-ph.CO].

[37] L. Miller, C. Heymans, T. Kitching, L. Van Waerbeke, T. Erben, et al., Mon.Not.Roy.Astron.Soc. 429, 2858 (2013), arXiv:1210.8201 [astro-ph.CO].

[38] J. Benjamin, L. Van Waerbeke, C. Heymans, M. Kilbinger, T. Erben, et al., Mon.Not.Roy.Astron.Soc. 431, 1547 (2013), arXiv:1212.3327 [astro-ph.CO],

[39] N. Benitez, Astrophys.J. 536, 571 (2000), arXiv:astro-ph/9811189 [astro-ph].

[40] M. Su, T. R. Slatyer, and D. P. Finkbeiner, Astrophys.J. 724, 1044 (2010), arXiv:1005.5480 [astro-ph.HE],

[41] M. Shirasaki and N. Yoshida, Astrophys.J. 786, 43 (2014), arXiv:1312.5032 [astro-ph.CO].

[42] S. Ando, E. Komatsu, T. Narumoto, and T. Totani, Mon.Not.Roy.Astron.Soc. 376, 1635 (2007), arXiv:astro-ph/0610155 [astro-ph].

[43] S. Ando and E. Komatsu, Phys.Rev. D87, 123539 (2013), arXiv:1301.5901 [astro-ph.CO],

[44] M. Bartelmann and P. Schneider, Phys.Rept. 340, 291 (2001) arXiv:astro-ph/9912508 [astro-ph]. 
[45] D. Munshi, P. Valageas, L. Van Waerbeke, and A. Heavens, Phys.Rept. 462, 67 (2008), arXiv:astro-ph/0612667 [astro-ph].

[46] D. N. Limber, Astrophys.J. 119, 655 (1954).

[47] N. Kaiser, Astrophys.J. 388, 272 (1992).

[48] R. de Putter and M. Takada, Phys.Rev. D82, 103522 (2010), arXiv:1007.4809 [astro-ph.CO].

[49] M. Oguri and M. Takada, Phys.Rev. D83, 023008 (2011), arXiv:1010.0744 [astro-ph.CO].

[50] A. Cooray and R. K. Sheth, Phys.Rept. 372, 1 (2002), arXiv:astro-ph/0206508 [astro-ph].

[51] R. K. Sheth and G. Tormen, Mon.Not.Roy.Astron.Soc. 308, 119 (1999), arXiv:astro-ph/9901122 [astro-ph].

[52] R. K. Sheth, H. Mo, and G. Tormen, Mon.Not.Roy.Astron.Soc. 323, 1 (2001), arXiv:astro-ph/9907024 [astro-ph].

[53] J. F. Navarro, C. S. Frenk, and S. D. White, Astrophys.J. 490, 493 (1997), arXiv:astro-ph/9611107 [astro-ph].

[54] G. Bryan and M. Norman, Astrophys.J. 495, 80 (1998), arXiv:astro-ph/9710107 [astro-ph].

[55] J. S. Bullock, T. S. Kolatt, Y. Sigad, R. S. Somerville, A. V. Kravtsov, et al., Mon.Not.Roy.Astron.Soc. 321, 559 (2001), arXiv:astro-ph/9908159 [astro-ph].

[56] L. Gao, C. Frenk, A. Jenkins, V. Springel, and S. White, Mon.Not.Roy.Astron.Soc. 419, 1721 (2012), arXiv:1107.1916 [astro-ph.CO],

[57] K. C. Y. Ng, R. Laha, S. Campbell, S. Horiuchi, B. Dasgupta, et al., (2013), arXiv:1310.1915 [astro-ph.CO].

[58] M. A. Sanchez-Conde and F. Prada, (2013), arXiv:1312.1729 [astro-ph.CO],

[59] G. Rodighiero, M. Vaccari, A. Franceschini, L. Tresse, O. L. Fevre, et al., aa 515, A8 (2010), arXiv:0910.5649 [astro-ph.CO].

[60] M. Ackermann et al. (Fermi-LAT Collaboration), Astrophys.J.Suppl. 203, 4 (2012), arXiv:1206.1896 [astro-ph.IM],

[61] A. Geringer-Sameth and S. M. Koushiappas, Phys.Rev.Lett. 107, 241303 (2011), arXiv:1108.2914 [astro-ph.CO].

[62] M. Ackermann et al. (Fermi-LAT collaboration), Phys.Rev.Lett. 107, 241302 (2011), arXiv:1108.3546 [astro-ph.HE],

[63] M. Ackermann et al. (Fermi-LAT Collaboration), Phys.Rev. D89, 042001 (2014), arXiv:1310.0828 [astro-ph.HE], 
[64] I. Cholis, D. Hooper, and S. D. McDermott, JCAP 1402,014 (2014), arXiv:1312.0608 [astro-ph.CO],

[65] S. Ando and D. Nagai, JCAP 1207, 017 (2012), arXiv:1201.0753 [astro-ph.HE].

[66] L. Goodenough and D. Hooper, (2009), arXiv:0910.2998 [hep-ph].

[67] D. Hooper and L. Goodenough, Phys.Lett. B697, 412 (2011), arXiv:1010.2752 [hep-ph].

[68] A. Boyarsky, D. Malyshev, and O. Ruchayskiy, Phys.Lett. B705, 165 (2011), arXiv:1012.5839 [hep-ph].

[69] D. Hooper and T. Linden, Phys.Rev. D84, 123005 (2011), arXiv:1110.0006 [astro-ph.HE],

[70] K. N. Abazajian and M. Kaplinghat, Phys.Rev. D86, 083511 (2012), arXiv:1207.6047 [astro-ph.HE],

[71] C. Gordon and O. Macias, Phys.Rev. D88, 083521 (2013), arXiv:1306.5725 [astro-ph.HE].

[72] K. N. Abazajian, N. Canac, S. Horiuchi, and M. Kaplinghat, (2014), arXiv:1402.4090 [astro-ph.HE],

[73] T. Daylan, D. P. Finkbeiner, D. Hooper, T. Linden, S. K. N. Portillo, et al., (2014), arXiv:1402.6703 [astro-ph.HE],

[74] J.-Q. Xia, A. Cuoco, E. Branchini, M. Fornasa, and M. Viel, Mon.Not.Roy.Astron.Soc. 416, 2247 (2011), arXiv:1103.4861 [astro-ph.CO].

[75] F. Prada, A. A. Klypin, A. J. Cuesta, J. E. Betancort-Rijo, and J. Primack, (2011), arXiv:1104.5130 [astro-ph.CO]. 